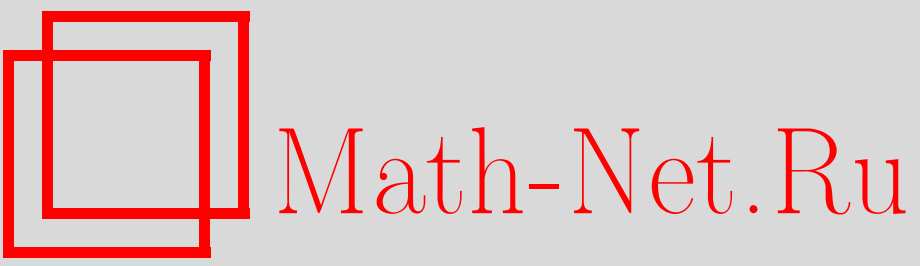

В. П. Маслов, Ультравторичное квантование и "духи" в квантованной энтропии, TMФ, 2001, том 129, номер 3, 464-490

DOI: https://doi.org/10.4213/tmf548

Использование Общероссийского математического портала Math-Net.Ru подразумевает, что вы прочитали и согласны с пользовательским соглашением

http://www.mathnet.ru/rus/agreement

Параметры загрузки:

IP: 54.198 .187 .58

26 апреля 2023 г., 09:15:01 
ТЕОРЕТИЧЕСКАЯ

И МАТЕМАТИЧЕСКАЯ

ФИЗИКА

Том 129, № 3

декабрь, 2001

(C) 2001 г.

В.П. Маслов*

\title{
УЛЬТРАВТОРИЧНОЕ КВАНТОВАНИЕ И “ДУ́ХИ" В КВАНТОВАННОЙ ЭНТРОПИИ
}

\begin{abstract}
Автором ранее была построена теория квантовой термодинамики, в которой сопряженным величинам - давлению и объему, температуре и энтропии, иначе говоря, сопряженным парам экстенсивных и интенсивных величин, аналогичных импульсам и координатам в классической механике, - сопоставляются операторы, подобно тому как это делается в квантовой механике. Показывается, что как в бозонном, так и в фермионном случаях квантованная энтропия, введенная как оператор в специальных пространствах Фока, содержащих новую переменную - так называемый статистический спин, зависит от некоторых переменных, которые не сказываются на физических результатах и поэтому названы автором "ду́хами".
\end{abstract}

Понятие квантованной энтропии и теория квантовой термодинамики рассматривались автором в ряде работ. Геометрическое квантование было проведено в работах [1]-[4], а обшее квантование подробно изложено в книге [5] и в статьях [6]-[22].

В настоящей статье мы покажем зависимость от некоторых несущественных переменных ультравторично-квантованной энтропии. Поскольку все понятия достаточно необычные и новые, вновь введем определения, пространства и операторы, которые здесь используются.

Особенно неожиданны в этой аксиоматике определения матрицы плотности и средних значений. Вместо выражения Гиббса $\operatorname{Sp}\left(e^{-\widehat{H} / k T}\right)$ рассматривается следуюшее выражение для свободной энергии: $\operatorname{Sp}\left(e^{-\widehat{F} / k T}\right)$, где $\widehat{F}$ - оператор свободной энергии. Казалось бы, здесь есть противоречие. Но дело в том, что следы берутся в разных пространствах. Однако все полученные таким образом результаты полностью согласуются со всеми известными положениями обычной статистической физики и термодинамики. Иначе говоря, это не противоречит старой теории, но, может быть, даст что-то сушественно новое.

Помимо координат точки могут быть еще другие степени свободы. Например, в классической механике это могут быть импульсы, а в квантовой теории - спины или еше какие-нибудь другие степени свободы. В излагаемой здесь теории добавляется еше одна степень свободы (минимальная) - просто номер, т.е. частице, кроме определенного места в пространстве, присваивается еше и номер.

* Московский государственный университет, Москва, Россия 
Эти номера можно интерпретировать как своеобразньй спин, принимаюший бесконечное множество значений. Будем называть его статистическим спином. Сам исходный гамильтониан не зависит от этого статистического спина. Однако уравнение Шредингера после ультравторичного квантования будет сушественно зависеть от фоковских пространств, учитывающих этот статистический спин [6]-[22], появятся также операторы рождения и уничтожения, которые связаны с этим статистическим спином или, другими словами, с этими номерами. Квантованная энтропия зависит от этого статистического спина и является оператором в пространстве Фока со статистическим спином. Эта процедура аналогична добавлению к уравнению Шредингера члена, включающего взаимодействие спина с постоянным магнитным полем, при этом получается дополнительная матриша в пространстве со спином (уравнение Паули). Оно уже будет сушественно зависеть от пространства со спином. Точно так же и ультравторично-квантованная энтропия, вводимая здесь, будет в этом пространстве являться оператором.

Квантование энтропии существенно меняет всю аксиоматику статистической физики и термодинамики. Оказывается, что давление и удельный объем являются не числами, а операторами. Причем коммутатор между ними имеет вид $1 / N$, где $N$ - число частиц. Иначе говоря, сопряженные термодинамические величины такие, как давление и объем, которые можно трактовать как импульсы и координаты, в квантовой термодинамике будут устроены так же, как в квантовой механике.

В термодинамике условия на термодинамический потенциал отвечают действию $\int p d q$, где $p$ - импульсы, т.е. объем и минус энтропия, $q$ - координаты, т.е. давление и температура. Аналогом импульсов являются все величины, которые могут быть удельными, иначе говоря экстенсивные величины, а аналогом координат являются интенсивные величины. В квантовой термодинамике, полученной с помощью квантования энтропии и представленной здесь аксиоматики, эти величины будут уже операторами. Например, оператор давления будет равен $\widehat{P}=(1 / N)(\partial / \partial v)$, где $v$ - удельный объем.

Оказывается, такая точка зрения дает возможность получить асимптотику вблизи точек возникновения новой фазы, в частности в точках возникновения трех фаз одновременно. Ответ дается с помощью функций Эйри или Вебера. Если же рассматривать логарифмический предел полученных выражений, то результатом как раз и будет обычная термодинамика. Однако поправки к главному члену в точках возникновения фаз оказываются довольно сушественными. Они аналогичны поправкам к логарифмической асимптотике в теории больших уклонений в фокальных точках [11], [23]. Предложенная автором концепция при больших $N$ и на специальных векторах в пространстве чисел заполнения совпадает с известными результатами. Например, мы получаем естественным образом уравнения Бардина-Купера-Шриффера (БКШ) и Гросса-Питаевского. При этом вводится квантованная энтропия и получаются уравнения, в которых учитывается еще и термодинамика. Эти уравнения новые, но тем не менее точка фазового перехода, получаюшаяся с их помощью, совпадает с полученной ранее физиками. Последующий переход к классической механике автоматически приводит к уравнениям для газа Ван-дер-Ваальса.

4 Теоретическая и математическая физика, т. 129, № 3, 2001 г. 
Таким образом, энтропия как бы "вбирает" в себя флуктуации. Рассматривается точное решение, которое и дает соотношение газа Ван-дер-Ваальса, а флуктуации не рассматриваются. Имеется в виду следуюшее: решение, например, уравнения Лиувилля можно трактовать как некоторую плотность распределения по координатам и импульсам. При этом плотность есть интеграл по импульсам, а значение скорости совпадает со значением момента. Замкнутой системы уравнений при этом не получается. Мы должны учитывать разность между средними значениями - дисперсию. Но если в уравнении Лиувилля рассматривать $\delta$-образное решение (в начальный момент берется $\delta$-функция на лагранжевом многообразии), то такой его вид сохранится и для такого распределения получится замкнутая система уравнений.

Для матрищы плотности Вигнера сушествует уравнение Вигнера. Если рассматривать $\delta$-образные решения уравнения Вигнера, то мы придем к задаче, которая фактически сводится к уравнению Шредингера, но записанному как система уравнений: для фазы $(\psi$-функции) и для ее модуля. Эта пара функций как раз и будет отвечать $\delta$-образному решению уравнения Вигнера. Для нахождения значений плотности и импульса получается замкнутая система уравнений. Она в точности совпадает с уравнением Шредингера, записанным вьшеуказанным образом.

По аналогии с уравнением Шредингера уравнение Вигнера переходит при $h \rightarrow 0$ в уравнение Лиувилля. А во что переходит само уравнение Шредингера? Чему оно отвечает? Мы видим, что уравнение Шредингера отвечает специальному решению уравнения Лиувилля - $\delta$-образному решению. Если начальное условие имеет вид $\delta(P(x)-$ $\left.P_{0}(x)\right) \varphi_{0}(x)$, то в момент времени $t \delta$-образный вид решения $\delta\left(P(x, t)-P_{0}(x)\right) \varphi(x, t)$ сохранится. Тогда получается замкнутая система уравнений для нахождения плотности, скорости и импульса. Таким образом, именно это решение уравнения Лиувилля аналогично решению уравнения Шредингера.

Оказывается, вводя квантованную энтропию, можно писать не само уравнение Шредингера, а уравнение для свободной энергии (иначе говоря, уравнение Шредингера, в которое входит квантованная энтропия, умноженная на температуру), а предельньй переход при $h \rightarrow 0$ приводит именно к уравнениям такого вида. То есть решению уравнения, аналогичного уравнению Лиувилля, соответствует точное $\delta$-образное решение нового уравнения - уравнения, содержашего коммутатор не с гамильтонианом $H$, являющимся внутренней энергией, а с гамильтонианом со свободной энергией, в который входит еше и температура. Оказывается, что не надо рассматривать флуктуации, так как подстановка $\delta$-образного решения приведет к уравнениям гидродинамики для газа Ван-дер-Ваальса.

Как известно, в элементарных формулах для "неравновесной энтропии" идеальных бозе- и ферми-газов [24] подсчитывается число вторичных распределений $N_{j}$ одинаковых частиц по $G_{j}$ состояниям. Далее оказывается, что окончательные ответы не зависят от $G_{j}$. Именно поэтому мы называем $G_{j}$ ду́хами. Если свободная энергия $F$ зависит от переменных, то в случае, когда эти переменные имеют физический смысл, производная $F$ по ним дает "сопряженную" термодинамическую величину. Если это не так, то мыназываем такую переменную "ду́хом”. С точки зрения нашего определения квантованной 
энтропии, так же как и в указанном подсчете состояний, “ду́хи” играют существенную роль.

Дело в том, что оператор квантованной энтропии мы определяем из двух условий: 1) $\operatorname{Sp}_{H}\left(e^{-\widehat{H} / \Theta}\right)$ и $\operatorname{Sp}_{F}\left(e^{-(\widehat{\widehat{H}} / \Theta)+\overline{\widehat{S}}}\right)$ должны совпадать (следы и операторы при этом рассматриваются, разумеется, в разных пространствах - в обычном фоковском пространстве $\mathcal{H}$ и в пространстве со статистическим спином); 2) в члене с энтропией должен возникать большой параметр в экспоненте, чтобы главный член следа совпадал с минимальным собственным значением оператора свободной энергии

$$
\overline{\widehat{F}}=\overline{\widehat{H}}-\Theta \overline{\widehat{S}}
$$

Этих условий недостаточно для однозначного определения оператора энтропии, отсюда и возникают лишние переменные - “духи". Но они не играют сушественной роли.

\section{1. БОЗОННЫЙ СЛУЧАЙ}

Введем пространства для рассмотрения $M$ парных частиц и $K$ частиц со статистическим спином (номером). Будем считать, что $N$ частиц находятся на трехмерном тоpe $\mathbf{T}$, длина сторон которого равна $L$, и частицы обладают спином $J$. Спиновую переменную будем обозначать буквой $\sigma$. Введем дискретную переменную $s$, которую будем называть статистическим спином (номером), для краткости будем употреблять сокращение статспин. Переменная $s$ принимает значения $1,2, \ldots$ Пусть $N=K+2 M$, где $K, M \geqslant 0$ - целые числа.

ОПРЕДЕЛЕНИЕ 1. Гильбертово пространство $\mathcal{F}_{K, M}$ состоит из функций

$$
\begin{gathered}
\Psi\left(x_{1}, \sigma_{1}, s_{1} ; x_{2}, \sigma_{2}, s_{2} ; \ldots ; x_{K}, \sigma_{K}, s_{K} ; y_{1}, \sigma_{1}^{\prime}, y_{2}, \sigma_{2}^{\prime}\right. \\
\left.y_{3}, \sigma_{3}^{\prime}, y_{4}, \sigma_{4}^{\prime} ; \ldots ; y_{2 M-1}, \sigma_{2 M-1}^{\prime}, y_{2 M}, \sigma_{2 M}^{\prime}\right), \\
x_{i}, y_{j} \in \mathbf{T}, \quad \sigma_{i}, \sigma_{j}^{\prime}=1,2, \ldots, 2 J+1, \quad i=1,2, \ldots, K, \quad j=1,2, \ldots, 2 M,
\end{gathered}
$$

симметричных относительно перестановок троек переменных $\left(x_{i}, \sigma_{i}, s_{i}\right)$ и $\left(x_{i^{\prime}}, \sigma_{i^{\prime}}, s_{i^{\prime}}\right)$, $i, i^{\prime}=1,2, \ldots, K$, и четверок переменных $\left(y_{2 j-1}, \sigma_{2 j-1}^{\prime}, y_{2 j}, \sigma_{2 j}^{\prime}\right)$ и $\left(y_{2 j^{\prime}-1}, \sigma_{2 j^{\prime}-1}^{\prime}, y_{2 j^{\prime}}\right.$, $\left.\sigma_{2 j^{\prime}}^{\prime}\right), j, j^{\prime}=1,2, \ldots, 2 M$. Кроме того, при фиксированных спиновых переменных $\sigma_{i}, \sigma_{j}^{\prime}$ и фиксированных статспиновых переменных $s_{i}, i=1,2, \ldots, K, j=1,2, \ldots, 2 M$, эти функции являются элементами пространства $L_{2}\left(\mathbf{T}^{N}\right)$, а также выполняется условие

$$
\begin{aligned}
& \sum_{\sigma_{1}=1}^{2 J+1} \ldots \sum_{\sigma_{K}=1}^{2 J+1} \sum_{\sigma_{1}^{\prime}=1}^{2 J+1} \ldots \sum_{\sigma_{2 M}=1}^{2 J+1} \sum_{s_{1}=1}^{\infty} \ldots \sum_{s_{K}=1}^{\infty} \int \ldots \int d x_{1} \ldots d x_{K} \times \\
& \quad \times d y_{1} d y_{2} \ldots d y_{2 M-1} d y_{2 M} \mid \Psi\left(x_{1}, \sigma_{1}, s_{1} ; x_{2}, \sigma_{2}, s_{2} ; \ldots ; x_{K}, \sigma_{K}, s_{K} ;\right. \\
& \left.y_{1}, \sigma_{1}^{\prime}, y_{2}, \sigma_{2}^{\prime} ; y_{3}, \sigma_{3}^{\prime}, y_{4}, \sigma_{4}^{\prime} ; \ldots ; y_{2 M-1}, \sigma_{2 M-1}^{\prime}, y_{2 M}, \sigma_{2 M}^{\prime}\right)\left.\right|^{2}<\infty
\end{aligned}
$$

Левая часть неравенства (2) равна квадрату нормы элемента (1) пространства $\mathcal{F}_{K, M}$. 
ОПРЕДЕЛЕНИЕ 2. Гильбертово пространство $\mathcal{F}=\bigoplus_{K, M=0}^{\infty} \mathcal{F}_{K, M}$ состоит из двухпараметрических последовательностей $\left\{\Psi_{K, M}\right\}, K, M=0,1, \ldots$, где член последовательности $\Psi_{K, M}$ является элементом пространства $\mathcal{F}_{K, M}$.

В силу симметрии функций $(1)$ пространство $\mathcal{F}$ является бозонным пространством Фока [25]. Вакуумный вектор пространства $\mathcal{F}$ имеет вид $\Psi_{0}=\left\{\Psi_{K, M}^{0}\right\}$, где $\Psi_{K, M}^{0}=0$ при $K>0$ или $M>0, \Psi_{0,0}^{0}=1$.

Стандартным образом [25] в пространстве $\mathcal{F}$ вводятся операторы рождения и уничтожения двух типов:

$\widehat{B}^{+}\left(y_{1}, \sigma_{1}^{\prime}, y_{2}, \sigma_{2}^{\prime}\right), \widehat{B}^{-}\left(y_{1}, \sigma_{1}^{\prime}, y_{2}, \sigma_{2}^{\prime}\right)-$ соответственно операторы рождения и уничтожения пар;

$\hat{b}^{+}(x, \sigma, s), \hat{b}^{-}(x, \sigma, s)-$ соответственно операторы рождения и уничтожения частиц со статспином.

Введем понятие ультравторичного квантования в бозонном случае.

Пусть $\mathcal{H}$ - бозонное пространство Фока, элементами которого являются последовательности $\left\{Y_{n}\right\}, \quad n=0,1, \ldots$, где член последовательности $Y_{n}$ является функцией $Y_{n}\left(x_{1}, \sigma_{1}, \ldots, x_{n}, \sigma_{n}\right)$ из пространства $L_{2}\left(\left(\mathbf{T}^{1} \times\{1, \ldots, 2 J+1\}\right)^{n}\right)$, симметричной относительно перестановок пар переменных $\left(x_{i}, \sigma_{i}\right)$ и $\left(x_{j}, \sigma_{j}\right), i, j=1,2, \ldots, n$. Операторы рождения и уничтожения в пространстве $\mathcal{H}$ будем обозначать соответственно $\psi^{+}(x, \sigma)$ и $\psi^{-}(x, \sigma)$.

Рассмотрим операторнозначный функционал $\hat{\rho}\left[b^{*}(\cdot), b(\cdot), B^{*}(\cdot), B(\cdot)\right]$, который зависит от функций $b(x, \sigma, s), B\left(y_{1}, \sigma_{1}, y_{2}, \sigma_{2}\right)$ таких, что

$$
\sum_{\sigma=1}^{2 J+1} \sum_{s=1}^{\infty} \int d x\left|b^{*}(x, \sigma, s)\right|^{2}<\infty, \quad \sum_{\sigma_{1}, \sigma_{2}=1}^{2 J+1} \iint d y_{1} d y_{2}\left|B\left(y_{1}, \sigma_{1}, y_{2}, \sigma_{2}\right)\right|^{2}<\infty
$$

и принимает значения в пространстве $\mathcal{H}$ :

$$
\begin{aligned}
\hat{\rho}\left[b^{*}(\cdot), b(\cdot), B^{*}(\cdot), B(\cdot)\right]=\sum_{K=0}^{\infty} \sum_{M=0}^{\infty} \frac{C_{K+2 M}}{K ! M !} \times \\
\quad \times\left(\sum_{\sigma_{1}, \sigma_{2}=1}^{2 J+1} \sum_{s=1}^{\infty} \iint d x_{1} d x_{2} b\left(x_{1}, \sigma_{1}, s\right) b^{*}\left(x_{1}, \sigma_{1}, s\right) \widehat{\psi}^{+}\left(x_{1}, \sigma_{1}\right) \widehat{\psi}^{-}\left(x_{2}, \sigma_{2}\right)\right)^{K} \times \\
\quad \times\left(\sum_{\sigma_{1}^{\prime}, \sigma_{2}^{\prime}=1}^{2 J+1} \iint d y_{1} d y_{2} B\left(y_{1}, \sigma_{1}^{\prime}, y_{2}, \sigma_{2}^{\prime}\right) \widehat{\psi}^{+}\left(y_{1}, \sigma_{1}^{\prime}\right) \widehat{\psi}^{+}\left(y_{2}, \sigma_{2}^{\prime}\right)\right)^{M} \times \\
\quad \times\left(\sum_{\sigma_{3}^{\prime}, \sigma_{4}^{\prime}=1}^{2 J+1} \iint d y_{3} d y_{4} B^{*}\left(y_{3}, \sigma_{3}^{\prime}, y_{4}, \sigma_{4}^{\prime}\right) \widehat{\psi}^{-}\left(y_{3}, \sigma_{3}^{\prime}\right) \widehat{\psi}^{-}\left(y_{4}, \sigma_{4}^{\prime}\right)\right)^{M} \times \\
\quad \times \exp \left(-\int d z \widehat{\psi}^{+}(z) \widehat{\psi}^{-}(z)\right),
\end{aligned}
$$

где $C_{N}, N=0,1,2, \ldots,-$ последовательность действительных положительных чисел такая, что $C_{N}=O\left(1 /(N !)^{\varepsilon}\right)$ при $N \rightarrow \infty, \varepsilon>0$, числа над операторами обозначают порядок действия этих операторов [26].

Пусть $\hat{A}$ - оператор в пространстве $\mathcal{H}$. 
ОПреДЕЛЕниЕ 3. Будем называть ультравторично-квантованным оператором $\overline{\hat{A}}$ в бозонном случае следующий оператор в пространстве $\mathcal{F}$ :

$$
\begin{aligned}
\overline{\hat{A}}= & A\left[\hat{b}^{+}(\cdot), \hat{b}^{-}(\cdot), \widehat{B}^{+}(\cdot), \widehat{B}^{-}(\cdot)\right] \exp \left(-\sum_{\sigma=1}^{2 J+1} \sum_{s=1}^{\infty} \int d x \hat{b}^{+}(x, \sigma, s) \hat{b}^{-}(x, \sigma, s)-\right. \\
& \left.-\sum_{\sigma_{1}^{\prime}, \sigma_{2}^{\prime}=1}^{2 J+1} \iint d y_{1} d y_{2} \widehat{B}^{+}\left(y_{1}, \sigma_{1}^{\prime}, y_{2}, \sigma_{2}^{\prime}\right) \widehat{B}^{-}\left(y_{1}, \sigma_{1}^{\prime}, y_{2}, \sigma_{2}^{\prime}\right)\right),
\end{aligned}
$$

где функционал $A\left[b^{*}(\cdot), b(\cdot), B^{*}(\cdot), B(\cdot)\right]$ выражается через $\hat{A}$ и $\hat{\rho}$ следующим образом:

$$
A\left[b^{*}(\cdot), b(\cdot), B^{*}(\cdot), B(\cdot)\right]=\operatorname{Sp}\left(\hat{\rho}\left[b^{*}(\cdot), b(\cdot), B^{*}(\cdot), B(\cdot)\right] \hat{A}\right) .
$$

1.1. Формальный переход к числам заполнения. Выберем полную ортонормированную в пространстве $L_{2}(\mathbf{T})$ систему функций $\left\{\varphi_{\alpha}(x)\right\}, \alpha=1,2, \ldots$ Рассмотрим следуюшую систему векторов в пространстве $\mathcal{F}$ :

$$
\begin{aligned}
\Psi_{\{n\},\{m\}}= & \left(\prod_{\alpha=1}^{\infty}\left(\prod_{\sigma=1}^{2 J+1}\left(\prod_{s=1}^{\infty}\left(\int d x \hat{b}^{+}(x, \sigma, s) \varphi_{\alpha}(x)\right)^{n_{\alpha \sigma s}} \frac{1}{\sqrt{n_{\alpha \sigma s} !}}\right)\right)\right) \times \\
& \times\left(\prod _ { \beta , \gamma = 1 } ^ { \infty } \left(\prod_{\sigma_{1}^{\prime}, \sigma_{2}^{\prime}=1}^{2 J+1}\left(\iint d y_{1} d y_{2} \varphi_{\beta}\left(y_{1}\right) \varphi_{\gamma}\left(y_{2}\right) \widehat{B}^{+}\left(y_{1}, \sigma_{1}^{\prime}, y_{2}, \sigma_{2}^{\prime}\right)\right)^{m_{\beta \gamma \sigma_{1}^{\prime} \sigma_{2}^{\prime}} \times}\right.\right. \\
& \left.\left.\times \frac{1}{\sqrt{m_{\beta \gamma \sigma_{1}^{\prime} \sigma_{2}^{\prime} !}}}\right)\right) \Psi_{0}
\end{aligned}
$$

где $\{n\}$ - набор целых чисел $n_{\alpha \sigma s} \geqslant 0$, зависящих от трех параметров $\alpha=1,2, \ldots, \sigma=$

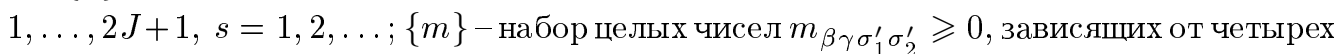
параметров $\beta, \gamma=1,2, \ldots, \sigma_{1}^{\prime}, \sigma_{2}^{\prime}=1, \ldots, 2 J+1$.

Система векторов (6) полная в пространстве $\mathcal{F}$.

Представлением ультравторично-квантованного оператора $\overline{\hat{A}}$ в числах заполнения является следующая матрица:

$$
A\left(\{n\},\{m\} ;\left\{n^{\prime}\right\},\left\{m^{\prime}\right\}\right)=\left(\Psi_{\{n\},\{m\}}, \overline{\hat{A}} \Psi_{\left\{n^{\prime}\right\},\left\{m^{\prime}\right\}}\right) .
$$

1.2. Символ ультравторичного квантования оператора. Символом оператора $\hat{A}$ вида (4) будем называть функционал (5). Имеет место следующая связь между представлением чисел заполнения и символом ультравторично-квантованного оператоpa.

Пусть наборы чисел $\{n\},\{m\},\left\{n^{\prime}\right\},\left\{m^{\prime}\right\}$ такие, что

$$
\begin{aligned}
& n_{\alpha \sigma s}=K \delta_{\alpha \alpha_{0}} \delta_{\sigma \sigma_{0}} \delta_{s s_{0}}, \quad m_{\beta \gamma \sigma \sigma^{\prime}}=M \delta_{\beta \beta_{0}} \delta_{\gamma \gamma_{0}} \delta_{\sigma \sigma_{1}} \delta_{\sigma^{\prime} \sigma_{2}} \\
& n_{\alpha \sigma s}^{\prime}=K \delta_{\alpha \alpha_{0}^{\prime}} \delta_{\sigma \sigma_{0}^{\prime}} \delta_{s s_{0}^{\prime}}, \quad m_{\beta \gamma \sigma \sigma^{\prime}}^{\prime}=M \delta_{\beta \beta_{0}^{\prime}} \delta_{\gamma \gamma_{0}^{\prime}} \delta_{\sigma \sigma_{1}^{\prime}} \delta_{\sigma^{\prime} \sigma_{2}^{\prime}}
\end{aligned}
$$


Рассмотрим функции

$$
\begin{aligned}
b_{1}(x, \sigma, s) & =\sum_{\alpha=1}^{\infty} \sqrt{n_{\alpha \sigma s}} \varphi_{\alpha}(x), \\
b_{2}(x, \sigma, s) & =\sum_{\alpha=1}^{\infty} \sqrt{n_{\alpha \sigma s}^{\prime}} \varphi_{\alpha}(x) \\
B_{1}\left(y_{1}, \sigma_{1}, y_{2}, \sigma_{2}\right) & =\sum_{\beta, \gamma=1}^{\infty} \sqrt{m_{\beta \gamma \sigma \sigma^{\prime}}} \varphi_{\beta}\left(y_{1}\right) \varphi_{\gamma}\left(y_{2}\right), \\
B_{2}\left(y_{1}, \sigma_{1}, y_{2}, \sigma_{2}\right) & =\sum_{\beta, \gamma=1}^{\infty} \sqrt{m_{\beta \gamma \sigma \sigma^{\prime}}^{\prime}} \varphi_{\beta}\left(y_{1}\right) \varphi_{\gamma}\left(y_{2}\right) .
\end{aligned}
$$

При $K, M \rightarrow \infty$ справедливо асимптотическое равенство

$$
A\left(\{n\},\{m\} ;\left\{n^{\prime}\right\},\left\{m^{\prime}\right\}\right) \sim A\left[b_{2}^{*}(\cdot), b_{1}(\cdot), B_{2}^{*}(\cdot), B_{1}(\cdot)\right] .
$$

1.3. Проекция ультравторично-квантованного оператора на $N$-частичное подпространство. Пусть числа $K_{0}, M_{0} \geqslant 0$ такие, что $N=K_{0}+2 M_{0}$. Рассмотрим в пространстве $\mathcal{F}$ подпространство, элементами которого являются двухпараметрические последовательности $\left\{\Psi_{K, M}\right\}$ такие, что

$$
\begin{aligned}
\Psi_{K, M} & =0, \quad K \neq K_{0} \text { или } M \neq M_{0}, \\
\Psi_{K_{0}, M_{0}} & =\Psi_{K_{0}, M_{0}}\left(x_{1}, \sigma_{1}, s_{1} ; \ldots ; x_{K_{0}}, \sigma_{K_{0}}, s_{K_{0}} ;\right. \\
& \left.y_{1}, \sigma_{1}^{\prime}, y_{2}, \sigma_{2}^{\prime} ; \ldots ; y_{2 M_{0}-1}, \sigma_{2 M_{0}-1}^{\prime}, y_{2 M_{0}}, \sigma_{2 M_{0}}^{\prime}\right)
\end{aligned}
$$

- функция, симметричная относительно перестановок пар переменных $\left(x_{i}, \sigma_{i}\right)$ и $\left(x_{i^{\prime}}, \sigma_{i^{\prime}}\right)$, симметричная относительно перестановок пар переменных $\left(y_{j}, \sigma_{j}^{\prime}\right)$ и $\left(y_{j^{\prime}}, \sigma_{j^{\prime}}^{\prime}\right)$ и симметричная относительно перестановок пар переменных $\left(x_{i}, \sigma_{i}\right)$ и $\left(y_{j}, \sigma_{j}^{\prime}\right), i, i^{\prime}=1, \ldots, K_{0}$, $j, j^{\prime}=1, \ldots, 2 M_{0}$. Обозначим это подпространство $\mathcal{F}_{K_{0}, M_{0}}^{\text {Symm }}$. Пусть оператор $\hat{A}$ в пространстве $\mathcal{H}$ такой, что вектор $\left\{Y_{n}\right\}$ он переводит в вектор $\left\{\widetilde{Y}_{n}\right\}$, где

$\widetilde{Y}_{n}\left(x_{1}, \ldots, x_{n}\right)=A_{n}\left(\stackrel{2}{x}_{1}, \ldots, \stackrel{2}{x}_{n},-i \frac{1}{\partial x_{1}}, \ldots,-i \frac{1}{\partial} \frac{1}{\partial x_{n}}\right) \widetilde{Y}_{n}\left(x_{1}, \ldots, x_{n}\right), \quad n=0,1,2, \ldots$

Имеет место следуюшая

ТЕОРема 1. Если $\Psi$ - әлемент подпространства $\mathcal{F}_{K_{0}, M_{0}}^{\mathrm{Symm}}$, то

$$
\begin{aligned}
& (\overline{\hat{A}} \Psi)_{K_{0}, M_{0}}=C_{N} \cdot N ! \cdot A_{N}\left(\stackrel{2}{x}_{1}, \ldots, \stackrel{2}{x}_{K_{0}}, \stackrel{1}{y}_{1}, \ldots, \stackrel{1}{y}_{2 M_{0}}\right. \\
& \left.-i \frac{1}{\partial x_{1}}, \ldots,-i \frac{\frac{1}{\partial}}{\partial x_{K_{0}}},-i \frac{\frac{1}{\partial}}{\partial y_{1}}, \ldots,-i \frac{\frac{1}{\partial}}{\partial y_{2} M_{0}}\right) \times \\
& \times \Psi_{K_{0}, M_{0}}\left(x_{1}, \sigma_{1}, s_{1} ; \ldots ; x_{K_{0}}, \sigma_{K_{0}}, s_{K_{0}} ;\right. \\
& \left.y_{1}, \sigma_{1}^{\prime}, y_{1}, \sigma_{2}^{\prime} ; \ldots ; y_{2 M_{0}-1}, \sigma_{2 M_{0}-1}^{\prime}, y_{2 M_{0}}, \sigma_{2 M_{0}}^{\prime}\right) \text {. }
\end{aligned}
$$


ДокАЗАТЕльСтво. Любой элемент $\Psi=\left\{\Psi_{K, M}\right\}$ пространства $\mathcal{F}$ представляется в виде [1]

$$
\begin{aligned}
\Psi= & \sum_{K=0}^{\infty} \sum_{M=0}^{\infty} \frac{1}{\sqrt{K ! M !}} \sum_{\sigma_{1}=0}^{2 J+1} \cdots \sum_{\sigma_{K}=0}^{2 J+1} \sum_{\sigma_{1}^{\prime}=0}^{2 J+1} \sum_{\sigma_{2}^{\prime}=0}^{2 J+1} \cdots \\
& \ldots \sum_{\sigma_{2 M-1}^{\prime}=0}^{2 J+1} \sum_{\sigma_{2 M}^{\prime}=0}^{2 J+1} \sum_{s_{1}=1}^{\infty} \ldots \sum_{s_{K}=0}^{\infty} \int \cdots \int d x_{1} \ldots d x_{K} d y_{1} \ldots d y_{2 M} \times \\
& \times \Psi_{K, M}\left(x_{1}, \sigma_{1}, s_{1} ; \ldots ; x_{K}, \sigma_{K}, s_{K} ; y_{1}, \sigma_{1}^{\prime}, y_{2}, \sigma_{2}^{\prime} ; \ldots ; y_{2 M-1}, \sigma_{2 M-1}^{\prime}, y_{2 M}, \sigma_{2 M}^{\prime}\right) \times \\
& \times \hat{b}^{+}\left(x_{1}, \sigma_{1}, s_{1}\right) \ldots \hat{b}^{+}\left(x_{K}, \sigma_{K}, s_{K}\right) \times \\
& \times \widehat{B}^{+}\left(y_{1}, \sigma_{1}^{\prime}, y_{2}, \sigma_{2}^{\prime}\right) \ldots \widehat{B}^{+}\left(y_{2 M-1}, \sigma_{2 M-1}^{\prime}, y_{2 M}, \sigma_{2 M}^{\prime}\right) \Psi_{0} .
\end{aligned}
$$

Кроме того, операторы

$$
\begin{aligned}
& \exp \left(-\sum_{\sigma=1}^{2 J+1} \sum_{s=1}^{\infty} \int d x \hat{b}^{+}(x, \sigma, s) \hat{b}^{-}(x, \sigma, s)-\right. \\
& \left.\quad-\sum_{\sigma_{1}^{\prime}, \sigma_{2}^{\prime}=1}^{2 J+1} \iint d y_{1} d y_{2} \widehat{B}^{+}\left(y_{1}, \sigma_{1}^{\prime}, y_{2}, \sigma_{2}^{\prime}\right) \widehat{B}^{-}\left(y_{1}, \sigma_{1}^{\prime}, y_{2}, \sigma_{2}^{\prime}\right)\right) \\
& \exp \left(-\int d z \widehat{\psi}^{+}(z) \widehat{\psi}^{-}(z)\right)
\end{aligned}
$$

являются ортогональными проекторами на вакуумный вектор соответственно в пространствах $\mathcal{F}$ и $\mathcal{H}[1]$.

Действуя на вектор (12) оператором (4) и учитывая свойства операторов (13) и операторов рождения и уничтожения $\widehat{\psi}^{ \pm}(x), \hat{b}^{ \pm}(x, \sigma, s), \widehat{B}^{ \pm}\left(y_{1}, \sigma_{1}^{\prime}, y_{2}, \sigma_{2}^{\prime}\right)$ :

$$
\begin{aligned}
{\left[\widehat{\psi}^{-}(x), \widehat{\psi}^{+}(y)\right] } & =\delta(x-y), \\
{\left[\hat{b}^{-}\left(x_{1}, \sigma_{1}, s_{1}\right), \hat{b}^{+}\left(x_{2}, \sigma_{2}, s_{2}\right)\right] } & =\delta_{\sigma_{1} \sigma_{2}} \delta_{s_{1} s_{2}} \delta\left(x_{1}-x_{2}\right), \\
{\left[\widehat{B}^{-}\left(y_{1}, \sigma_{1}^{\prime}, y_{2}, \sigma_{2}^{\prime}\right), \widehat{B}^{+}\left(y_{3}, \sigma_{3}^{\prime}, y_{4}, \sigma_{4}^{\prime}\right)\right] } & =\delta_{\sigma_{1}^{\prime} \sigma_{3}^{\prime}} \delta_{\sigma_{2}^{\prime} \sigma_{4}^{\prime}} \delta\left(y_{1}-y_{3}\right) \delta\left(y_{2}-y_{4}\right),
\end{aligned}
$$

где остальные коммутаторы равны нулю,

$$
\widehat{\psi}^{-}(x) Y_{0}=0, \quad \hat{b}^{-}(x, \sigma, s) \Psi_{0}=0, \quad \widehat{B}^{-}\left(y_{1}, \sigma_{1}^{\prime}, y_{2}, \sigma_{2}^{\prime}\right) \Psi_{0}=0,
$$

получим утверждение теоремы.

Из теоремы 1 следует, что оператор $\overline{\hat{A}}$ на подпространстве $\mathcal{F}_{K, M}$ зависит от $N=K+$ $2 M$ и не зависит от $K$ и $M$ в отдельности.

Из теоремы 1 также следует соответствие алгебр операторов $A, \hat{A}, \overline{\hat{A}}$.

Пусть $\psi_{N}$ - элемент $N$-частичного подпространства. В пространстве $\mathcal{F}$ ему отвечает последовательность элементов $\left\{\widetilde{\psi}_{N}\right\}$ таких, что $\widetilde{\psi}_{N^{\prime}}=0$ при $N^{\prime} \neq N$ и $\widetilde{\psi}_{N}=\psi_{N}$. В силу теоремы 1 имеет место равенство $\overline{\hat{A}}\left\{\widetilde{\psi}_{N}\right\}=\left\{A_{N} \widetilde{\psi}_{N}\right\}$. 
Значит,

$$
\overline{\hat{A}}^{2}\left\{\widetilde{\psi}_{N}\right\}=\overline{\hat{A}}\left\{A_{N} \widetilde{\psi}_{N}\right\}=\left\{A_{N}^{2} \widetilde{\psi}_{N}\right\} .
$$

Очевидно, что

$$
f\left(\overline{\hat{A}}\left\{\widetilde{\psi}_{N}\right\}\right)=\left\{f\left(A_{N}\right) \widetilde{\psi}_{N}\right\} .
$$

Аналогично для $f(\hat{A})$.

\section{2. ФЕРМИОННЫЙ СЛУЧАЙ}

Введем понятие ультравторичного квантования в фермионном случае. Пусть $\mathcal{H}-$ фермионное пространство Фока, элементами которого являются последовательности $\left\{Y_{n}\right\}, n=0,1, \ldots$, где член последовательности $Y_{n}$ является функцией $Y_{n}\left(x_{1}, \sigma_{1}, \ldots\right.$ $\left.\ldots, x_{n}, \sigma_{n}\right)$ из пространства $L_{2}\left(\left(\mathbf{T}^{1} \times\{1, \ldots, 2 J+1\}\right)^{n}\right)$, антисимметричной относительно перестановок пар переменных $\left(x_{i}, \sigma_{i}\right)$ и $\left(x_{j}, \sigma_{j}\right), i, j=1,2, \ldots, n$. Операторы рождения и уничтожения в пространстве $\mathcal{H}$ будем обозначать соответственно $\psi^{+}(x, \sigma)$ и $\psi^{-}(x, \sigma)$.

Рассмотрим операторнозначный функционал $\hat{\rho}\left[b^{*}(\cdot), b(\cdot), B^{*}(\cdot), B(\cdot)\right]$, который зависит от функций $b(x, \sigma, s), B\left(y_{1}, \sigma_{1}, y_{2}, \sigma_{2}\right)$ таких, что

$$
\sum_{\sigma=1}^{2 J+1} \sum_{s=1}^{\infty} \int d x\left|b^{*}(x, \sigma, s)\right|^{2}<\infty, \quad \sum_{\sigma_{1}, \sigma_{2}=1}^{2 J+1} \iint d y_{1} d y_{2}\left|B\left(y_{1}, \sigma_{1}, y_{2}, \sigma_{2}\right)\right|^{2}<\infty
$$

и принимает значения в пространстве $\mathcal{H}$ :

$$
\begin{aligned}
\hat{\rho}\left[b^{*}(\cdot),\right. & \left.b(\cdot), B^{*}(\cdot), B(\cdot)\right]=\sum_{K=0}^{\infty} \sum_{M=0}^{\infty} \frac{C_{K+2 M}}{K ! M !} \times \\
& \times\left(\sum_{\sigma_{1}, \sigma_{2}=1}^{2 J+1} \iint d y_{1} d y_{2} B\left(y_{1}, \sigma_{1}^{\prime}, y_{2}, \sigma_{2}^{\prime}\right) \widehat{\psi}^{+}\left(y_{1}, \sigma_{1}^{\prime}\right) \widehat{\psi}^{+}\left(y_{2}, \sigma_{2}^{\prime}\right)\right)^{M} \times \\
& \times \sum_{\sigma_{1}=1}^{2 J+1} \cdots \sum_{\sigma_{K}=1}^{2 J+1} \sum_{\sigma_{K+1}=1}^{2 J+1} \cdots \sum_{\sigma_{2 K}=1}^{2 J+1} \sum_{s_{1}=1}^{\infty} \cdots \sum_{s_{K}=1}^{\infty} \iint d x_{1} \ldots d x_{K} d x_{K+1} \ldots d x_{2 K} \times \\
& \times \widehat{\psi}^{+}\left(x_{1}, \sigma_{1}\right) b\left(x_{1}, \sigma_{1}, s_{1}\right) \ldots \widehat{\psi}^{+}\left(x_{K}, \sigma_{K}\right) b\left(x_{K}, \sigma_{K}, s_{K}\right) \times \\
& \times \widehat{P}_{0} b^{*}\left(x_{2 K}, \sigma_{2 K}, s_{K}\right) \widehat{\psi}^{-}\left(x_{2 K}, \sigma_{2 K}\right) \ldots b^{*}\left(x_{K+1}, \sigma_{K+1}, s_{1}\right) \widehat{\psi}^{-}\left(x_{K+1}, \sigma_{K+1}\right) \times \\
& \times\left(\sum_{\sigma_{3}^{\prime}, \sigma_{4}^{\prime}=1}^{2 J+1} \iint d y_{3} d y_{4} B^{*}\left(y_{3}, \sigma_{2}^{\prime}, y_{4}, \sigma_{4}^{\prime}\right) \widehat{\psi}^{-}\left(y_{4}, \sigma_{4}\right) \widehat{\psi}^{-}\left(y_{3}, \sigma_{3}\right)\right),
\end{aligned}
$$

где $C_{N}, N=0,1,2, \ldots,-$ последовательность действительных положительных чисел, удовлетворяющая при $N \rightarrow \infty$ оценке $C_{N}=O\left(1 /(N !)^{\varepsilon}\right)$ для некоторого $\varepsilon>0, \widehat{P}_{0}-$ ортогональный проектор на вакуумный вектор в пространстве $\mathcal{H}$.

Пусть $\hat{A}$ - оператор в пространстве $\mathcal{H}$. 
ОПрЕДЕлЕниЕ 4. Будем называть ультравторично-квантованным оператором $\overline{\hat{A}}$ в фермионном случае оператор вида (4) в пространстве $\mathcal{F}$, в котором функционал $A\left[b^{*}(\cdot)\right.$, $\left.b(\cdot), B^{*}(\cdot), B(\cdot)\right]$ выражается через $\hat{A}$ по формуле (5), а $\hat{\rho}$ имеет вид (16).

2.1. Числа заполнения и символ ультравторично-квантованного оператора. Формальный переход к числам заполнения в фермионном случае производится совершенно аналогично бозонному случаю (см. п. 1.1).

Символ ультравторично-квантованного оператора в фермионном случае определяется так же, как в бозонном, и для него справедливо асимптотическое равенство, аналогичное (8).

В случае произвольных наборов чисел заполнения имеет место следуюшее тождество, справедливое также и для бозонов:

$$
\begin{aligned}
& A\left(\{n\},\{m\} ;\left\{n^{\prime}\right\},\left\{m^{\prime}\right\}\right)=\left(\prod_{\alpha=1}^{\infty} \prod_{\sigma=1}^{2 J+1} \prod_{s=1}^{\infty} \sqrt{n_{\alpha \sigma s} ! n_{\alpha \sigma s}^{\prime} !}\right) \times \\
& \times\left(\prod_{\beta, \gamma=1}^{\infty} \prod_{\sigma_{1}^{\prime}, \sigma_{2}^{\prime}=1}^{2 J+1} \sqrt{m_{\beta \gamma \sigma_{1}^{\prime} \sigma_{2}^{\prime} ! m_{\beta \gamma \sigma_{1}^{\prime} \sigma_{2}^{\prime}}^{\prime}}}\right) \times \\
& \times \int \cdots \int\left(\prod_{\alpha=1}^{\infty} \prod_{\sigma=1}^{2 J+1} \prod_{s=1}^{\infty} \frac{d z_{\alpha \sigma s}}{2 \pi i\left(z_{\alpha \sigma s}\right)^{n_{\alpha \sigma s}+1}} \frac{d z_{\alpha \sigma s}^{\prime}}{2 \pi i\left(z_{\alpha \sigma s}^{\prime}\right)^{n_{\alpha \sigma s}^{\prime}+1}}\right) \times
\end{aligned}
$$

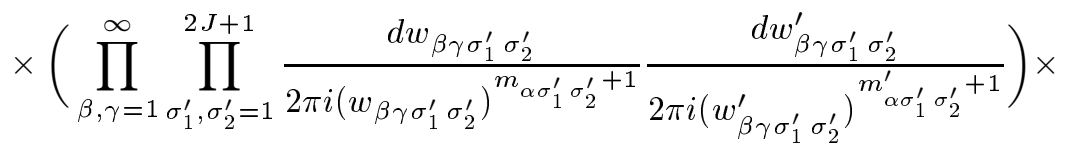

$$
\begin{aligned}
& \times A\left[b_{2}^{*}(\cdot), b_{1}(\cdot), B_{2}^{*}(\cdot), B_{1}(\cdot)\right],
\end{aligned}
$$

где однократные интегралы по переменным $z_{\alpha \sigma s}, z_{\alpha \sigma s}^{\prime}, w_{\beta \gamma \sigma_{1}^{\prime} \sigma_{2}^{\prime}}, w_{\beta \gamma \sigma_{1}^{\prime} \sigma_{2}^{\prime}}$ берутся в комплексной плоскости по любому контуру $C$, обходящему нулевую точку против часовой стрелки, а функции $b_{2}(x, \sigma, s), b_{1}(x, \sigma, s), B_{1}\left(y_{1}, \sigma_{1}^{\prime}, y_{2}, \sigma_{2}^{\prime}\right), B_{2}\left(y_{1}, \sigma_{1}^{\prime}, y_{2}, \sigma_{2}^{\prime}\right)$ зависят от $z_{\alpha \sigma s}, z_{\alpha \sigma s}^{\prime}, w_{\beta \gamma \sigma_{1}^{\prime} \sigma_{2}^{\prime}}, w_{\beta \gamma \sigma_{1}^{\prime} \sigma_{2}^{\prime}}^{\prime}$ следуюшим образом:

$$
\begin{aligned}
b_{2}(x, \sigma, s) & =\sum_{\alpha=1}^{\infty} \varphi_{\alpha}(x) z_{\alpha \sigma s}, \\
b_{1}(x, \sigma, s) & =\sum_{\alpha=1}^{\infty} \varphi_{\alpha}(x)\left(z_{\alpha \sigma s}^{\prime}\right)^{*}, \\
B_{1}\left(y_{1}, \sigma_{1}, y_{2}, \sigma_{2}\right) & =\sum_{\beta, \gamma=1}^{\infty} \varphi_{\beta}\left(y_{1}\right) \varphi_{\gamma}\left(y_{2}\right) w_{\beta \gamma \sigma_{1}^{\prime} \sigma_{2}^{\prime}}, \\
B_{2}\left(y_{1}, \sigma_{1}^{\prime}, y_{2}, \sigma_{2}^{\prime}\right) & =\sum_{\beta, \gamma=1}^{\infty} \varphi_{\beta}\left(y_{1}\right) \varphi_{\gamma}\left(y_{2}\right)\left(w_{\beta \gamma \sigma_{1}^{\prime} \sigma_{2}^{\prime}}\right)^{*} .
\end{aligned}
$$


2.2. Проекция ультравторично-квантованного оператора на $N$-частичное подпространство. Пусть числа $K_{0}, M_{0} \geqslant 0$ такие, что $N=K_{0}+2 M_{0}$. Рассмотрим в пространстве $\mathcal{F}$ подпространство, элементами которого являются двухпараметрические последовательности $\left\{\Psi_{K, M}\right\}$ вида (9), в которых функция $\psi_{K_{0}, M_{0}}$ антисимметрична относительно перестановок пар переменных $\left(x_{i}, \sigma_{i}\right)$ и $\left(x_{i^{\prime}}, \sigma_{i^{\prime}}\right)$, антисимметрична относительно перестановок пар переменных $\left(y_{j}, \sigma_{j}^{\prime}\right)$ и $\left(y_{j^{\prime}}, \sigma_{j^{\prime}}^{\prime}\right)$ и антисимметрична относительно перестановок пар переменных $\left(x_{i}, \sigma_{i}\right)$ и $\left(y_{j}, \sigma_{j}^{\prime}\right), \quad i, i^{\prime}=1, \ldots, K_{0}, \quad j, j^{\prime}=$ $1, \ldots, 2 M_{0}$. Обозначим это подпространство $\mathcal{F}_{K_{0}, M_{0}}^{\text {Asymm }}$. Пусть оператор $\hat{A}$ в пространстве $\mathcal{H}$ такой, что вектор $\left\{Y_{n}\right\}$ он переводит в вектор $\left\{\widetilde{Y}_{n}\right\}(10)$.

Имеет место следующая

ТЕОрема 2. Если $\Psi$ - әлемент подпространства $\mathcal{F}_{K_{0}, M_{0}}^{\text {Asymm }}$, то выполняется равенство (11).

ДоказАТЕЛЬСтво. Любой элемент $\Psi=\left\{\Psi_{K, M}\right\}$ пространства $\mathcal{F}$ имеет вид (12) [1]. Кроме того, операторы

$$
\begin{aligned}
\exp ( & -\sum_{\sigma=1}^{2 J+1} \sum_{s=1}^{\infty} \int d x \hat{b}^{+}(x, \sigma, s) \hat{b}^{-}(x, \sigma, s)- \\
& \left.-\sum_{\sigma_{1}^{\prime}, \sigma_{2}^{\prime}=1}^{2 J+1} \iint d y_{1} d y_{2} \widehat{B}^{+}\left(y_{1}, \sigma_{1}^{\prime}, y_{2}, \sigma_{2}^{\prime}\right) \widehat{B}^{-}\left(y_{1}, \sigma_{1}^{\prime}, y_{2}, \sigma_{2}^{\prime}\right)\right)
\end{aligned}
$$

и $\widehat{P}_{0}$ являются ортогональными проекторами на вакуумный вектор соответственно в пространствах $\mathcal{F}$ и $\mathcal{H}[1]$.

Действуя на вектор $\psi$ оператором $\overline{\hat{A}}$ и учитывая свойства операторов (18) и $\widehat{P}_{0}$ и свойства (14), (15) операторов рождения и уничтожения $\widehat{\psi}^{ \pm}(x), \hat{b}^{ \pm}(x, \sigma, s), \widehat{B}^{ \pm}\left(y_{1}, \sigma_{1}^{\prime}\right.$, $\left.y_{2}, \sigma_{2}^{\prime}\right)$, получим утверждение теоремы.

\section{3. МАТРИЦА ПЛОТНОСТИ ДЛЯ ФЕРМИОНОВ}

3.1. Случай отсутствия операторов рождения-уничтожения пар $\widehat{B}^{+}, \widehat{B}^{-}$. Рассмотрим уравнение для матрицы плотности системы $N$ фермионов

$$
i \frac{\partial \rho}{\partial t}\left(x_{1}, y_{1} ; \ldots ; x_{N}, y_{N} ; t\right)=\left(H_{N}(x)-H_{N}(y)\right) \rho\left(x_{1}, y_{1} ; \ldots ; x_{N}, y_{N} ; t\right)
$$

где $H_{N}(x)$ - оператор в пространстве $L_{2}\left(\mathbf{T}^{N}\right)$ вида

$$
\begin{aligned}
H_{N}(x) \Phi\left(x_{1}, \ldots, x_{N}\right)= & \int d y\left(T\left(x_{1}, y\right) \Phi\left(y, x_{2}, \ldots, x_{N}\right)+\right. \\
& +T\left(x_{2}, y\right) \Phi\left(x_{1}, y, x_{3}, \ldots, x_{N}\right)+\cdots \\
& \left.\cdots+T\left(x_{N}, y\right) \Phi\left(x_{1}, x_{2}, \ldots, x_{N-1}, y\right)\right)+ \\
& +\iint d z d w\left(V\left(x_{1}, x_{2} ; z, w\right) \Phi\left(z, w, x_{3}, \ldots, x_{N-1}, x_{N}\right)+\cdots\right. \\
& \left.\cdots+V\left(x_{N-1}, x_{N} ; z, w\right) \Phi\left(x_{1}, x_{2}, x_{3}, \ldots, x_{N-2}, z, w\right)\right),
\end{aligned}
$$


а функция $\rho\left(x_{1}, y_{1} ; \ldots ; x_{N}, y_{N} ; t\right)$ является антисимметричной относительно перестановок переменных $x_{j}$ и $x_{k}$ и антисимметричной относительно перестановок переменных $y_{j}$ и $y_{k}, j, k=1, \ldots, N$, и удовлетворяет условиям

$$
\begin{gathered}
\rho\left(x_{1}, y_{1} ; \ldots ; x_{N}, y_{N} ; t\right)=\rho^{*}\left(y_{1}, x_{1} ; \ldots ; y_{N}, x_{N} ; t\right), \\
\int \cdots \int d x_{1} \ldots d x_{N} \rho\left(x_{1}, x_{1} ; \ldots ; x_{N}, x_{N} ; t\right)<\infty .
\end{gathered}
$$

В силу антисимметрии функции $\rho\left(x_{1}, y_{1} ; \ldots ; x_{N}, y_{N} ; t\right)$ относительно переменных $x$ и переменных $y$ эта функция симметрична относительно перестановок пар переменных $\left(x_{j}, y_{j}\right)$ и $\left(x_{k}, y_{k}\right), j, k=1, \ldots, N$. Поэтому к уравнению (20) можно применить процедуру ультравторичного квантования, которая немного отличается от обычной.

Введем пространство $\mathcal{L}_{N}$, элементами которого являются функции

$$
\Phi\left(x_{1}, y_{1} ; \ldots ; x_{N}, y_{N}\right) \in L_{2}\left(\mathbf{T}^{2 N}\right)
$$

симметричные относительно перестановок пар переменных $\left(x_{j}, y_{j}\right)$ и $\left(x_{k}, y_{k}\right), j, k=$ $1, \ldots, N$. Рассмотрим пространство $\mathcal{L}=\bigoplus_{N=0}^{\infty} \mathcal{L}_{N}$, которое состоит из последовательностей $\left\{\Phi_{N}\right\}, \quad N=0,1, \ldots$, функций $\Phi_{N} \in \mathcal{L}_{N}$. Пространство $\mathcal{L}$ является бозонным фоковским, и в нем можно ввести операторы рождения $\hat{b}^{+}(x, y)$ и уничтожения $\hat{b}^{-}(x, y)$, а также вакуумный вектор $\Phi_{0}$ такие, что

$$
\begin{aligned}
{\left[\hat{b}^{-}(x, y), \hat{b}^{+}\left(x^{\prime}, y^{\prime}\right)\right] } & =\delta\left(x-x^{\prime}\right) \delta\left(y-y^{\prime}\right), \\
{\left[\hat{b}^{ \pm}(x, y), \hat{b}^{ \pm}\left(x^{\prime}, y^{\prime}\right)\right] } & =0, \\
\hat{b}^{-}(x, y) \Phi_{0} & =0, \quad\left(\Phi_{0}, \Phi_{0}\right)=1 .
\end{aligned}
$$

Рассмотрим в пространстве $\mathcal{L}$ следуюшие операторы:

$$
\begin{aligned}
\overline{\widehat{H}}= & \sum_{N=0}^{\infty} \int \ldots \int d x_{1} d y_{1} \ldots d x_{N} d y_{N} \hat{b}^{+}\left(x_{1}, y_{1}\right) \ldots \hat{b}^{+}\left(x_{N}, y_{N}\right) \times \\
& \times\left(H_{N}(x)-H_{N}(y)\right) \underset{x_{1}, \ldots, x_{N}}{\operatorname{Asymm}}\left(\hat{b}^{-}\left(x_{1}, y_{1}\right) \ldots \hat{b}^{-}\left(x_{N}, y_{N}\right)\right) \times \\
& \times \exp \left(-\iint d z d w \hat{b}^{+}(z, w) \hat{b}^{-}(z, w)\right), \\
\overline{\widehat{E}}= & \sum_{N=0}^{\infty} \int \cdots \int d x_{1} d y_{1} \ldots d x_{N} d y_{N} \hat{b}^{+}\left(x_{1}, y_{1}\right) \ldots \hat{b}^{+}\left(x_{N}, y_{N}\right) \times \\
& \times \operatorname{Asymm}_{x_{1}, \ldots, x_{N}}\left(\hat{b}^{-}\left(x_{1}, y_{1}\right) \ldots \hat{b}^{-}\left(x_{N}, y_{N}\right)\right) \times \\
& \times \exp \left(-\iint d z d w \hat{b}^{+}(z, w) \hat{b}^{-}(z, w)\right),
\end{aligned}
$$




$$
\begin{aligned}
\widehat{\widehat{N}}= & \sum_{N=0}^{\infty} N \int \ldots \int d x_{1} d y_{1} \ldots d x_{N} d y_{N} \hat{b}^{2}\left(x_{1}, y_{1}\right) \ldots \hat{b}^{+}\left(x_{N}, y_{N}\right) \times \\
& \times \underset{x_{1}, \ldots, x_{N}}{\operatorname{Asymm}}\left(\hat{b}^{-}\left(x_{1}, y_{1}\right) \ldots \hat{b}^{-}\left(x_{N}, y_{N}\right)\right) \times \\
& \times \exp \left(-\iint d z d w \hat{b}^{+}(z, w) \hat{b}^{-}(z, w)\right)
\end{aligned}
$$

где $\operatorname{Asymm}_{x_{1}, \ldots, x_{N}}$ - оператор антисимметризации по переменным $x_{1}, \ldots, x_{N}$. Оператор $\overline{\widehat{H}}$ является ультравторично-квантованным гамильтонианом уравнения для матрицы плотности $(20), \overline{\widehat{N}}$ - ультравторично-квантованный оператор числа частиц, $\widehat{\widehat{E}}$ ультравторично-квантованный единичный оператор. Имеет место

ПРЕДЛОЖЕНИЕ 1. Пусть $\Phi(t)$ - функиия аргумента $t \in \mathbb{R}$ со значением в $\mathcal{L}$, которая удовлетворяет уравнениям

$$
i \overline{\widehat{E}} \frac{\partial}{\partial t} \Phi(t)=\overline{\widehat{H}} \Phi(t), \quad \overline{\widehat{N}} \Phi(t)=N \overline{\widehat{E}} \Phi(t), \quad \overline{\widehat{E}} \Phi(t) \neq 0
$$

\section{Тогда функиия}

$$
\Phi_{N}\left(x_{1}, y_{1} ; \ldots ; x_{N}, y_{N} ; t\right)=\left(\Phi_{0}, \hat{b}^{-}\left(x_{1}, y_{1}\right) \ldots \hat{b}^{-}\left(x_{N}, y_{N}\right) \overline{\widehat{E}} \Phi(t)\right)
$$

является решением уравнения (20).

Рассмотрим случай, когда коммутаторы (14) можно считать малыми. Тогда асимптотика решений задачи (27) выражается через периодические решения гамильтоновой системы, отвечающей гамильтониану

$$
\begin{aligned}
\mathcal{H}\left(b^{*}(\cdot), b(\cdot)\right)= & \left(\sum_{N=0}^{\infty} \int \ldots \int d x_{1} d y_{1} \ldots d x_{N} d y_{N} b^{*}\left(x_{1}, y_{1}\right) \ldots b^{*}\left(x_{N}, y_{N}\right) \times\right. \\
& \left.\times\left(H_{N}(x)-H_{N}(y)\right) \underset{x_{1}, \ldots, x_{N}}{\operatorname{Asymm}}\left(b\left(x_{1}, y_{1}\right) \ldots b\left(x_{N}, y_{N}\right)\right)\right) \times \\
& \times\left(\sum_{N=0}^{\infty} \int \cdots \int d x_{1} d y_{1} \ldots d x_{N} d y_{N} b^{*}\left(x_{1}, y_{1}\right) \ldots b^{*}\left(x_{N}, y_{N}\right) \times\right. \\
& \left.\times \underset{x_{1}, \ldots, x_{N}}{\operatorname{Asymm}}\left(b\left(x_{1}, y_{1}\right) \ldots b\left(x_{N}, y_{N}\right)\right)\right)^{-1} \cdot
\end{aligned}
$$

Асимптотика решений уравнений (27) таких, что

$$
\Phi_{N}^{*}\left(x_{1}, y_{1} ; \ldots ; x_{N}, y_{N} ; t\right)=\Phi_{N}\left(y_{1}, x_{1} ; \ldots ; y_{N}, x_{N} ; t\right)
$$


может быть выражена через решения гамильтоновой системы, для которых справедливо $b(x, y, t)=b^{*}(y, x, t)$. Система уравнений Гамильтона при таком условии принимает вид

$$
\begin{aligned}
i \frac{\partial}{\partial t} G\left(x_{1}, x_{2}\right)= & \int d z T\left(x_{2}, z\right) G\left(x_{1}, z\right)-\int d z T\left(z, x_{1}\right) G\left(z, x_{2}\right)+ \\
& +\iiint d y d z d w V\left(x_{2}, y, z, w\right)\left(G\left(x_{1}, z\right) G^{*}(y, w)-G\left(x_{1}, w\right) G^{*}(y, z)\right)- \\
& -\iiint d y d z d w V\left(z, w, x_{1}, y\right)\left(G\left(z, x_{2}\right) G(w, y)-G\left(w, x_{2}\right) G(z, y)\right),(29)
\end{aligned}
$$

где функция $G(x, y)$ выражается через функцию $b(x, y)$ как решение уравнения

$$
G(x, y)=\int d z b(x, z) b^{*}(y, z)+\iint d z d w b(x, z) b^{*}(w, z) G(w, y)
$$

3.2. Учет операторов $B^{+}, B^{-}$и температурные уравнения БКШ-Боголюбова. Ультравторичное квантование уравнения (20) можно провести с учетом пар. В этом случае в пространстве $\mathcal{L}$ появятся операторы $\widehat{B}_{1}^{ \pm}\left(x_{1}, x_{2}\right)$ и $\widehat{B}_{2}^{ \pm}\left(x_{1}, x_{2}\right)$, а операторы $\widehat{\widehat{H}}, \widehat{\widehat{E}}$ примут вид

$$
\begin{aligned}
\overline{\hat{H}}= & \sum_{k, m=0}^{\infty} \int \ldots \int d x_{1} d y_{1} \ldots d x_{k+2 m} d y_{k+2 m} \hat{b}^{+}\left(x_{1}, y_{1}\right) \ldots \hat{b}^{+}\left(x_{k}, y_{k}\right) \times \\
& \times \widehat{B}_{1}^{+}\left(x_{k+1}, x_{k+2}\right) \widehat{B}_{2}^{+}\left(y_{k+1}, y_{k+2}\right) \ldots \widehat{B}_{1}^{+}\left(x_{k+2 m-1}, x_{k+2 m}\right) \widehat{B}_{2}^{+}\left(y_{k+2 m-1}, y_{k+2 m}\right) \times \\
& \times\left(H_{k+2 m}(x)-H_{k+2 m}(y)\right) \times \\
& \times \underset{x_{1}, \ldots, x_{k+2 m}}{\operatorname{Asymm}} y_{1}, \ldots, y_{k+2 m}\left(\hat{b}^{-}\left(x_{1}, y_{1}\right) \ldots \hat{b}^{-}\left(x_{k}, y_{k}\right) \widehat{B}_{1}^{-}\left(x_{k+1}, x_{k+2}\right) \times\right. \\
& \left.\times \widehat{B}_{2}^{-}\left(y_{k+1}, y_{k+2}\right) \ldots \widehat{B}_{1}^{-}\left(x_{k+2 m-1}, x_{k+2 m}\right) \widehat{B}_{2}^{-}\left(y_{k+2 m-1}, y_{k+2 m}\right)\right) \times \\
& \times \exp \left(-\iint d x d y \hat{b}^{+}(x, y) \hat{b}^{-}(x, y)-\iint d z d w \widehat{B}_{1}^{+}(z, w) \widehat{B}_{1}^{-}(z, w)-\right. \\
& \left.-\iint^{2} d z^{\prime} d w^{\prime} \widehat{B}_{2}^{+}\left(z^{\prime}, w^{\prime}\right) \widehat{B}_{2}^{-}\left(z^{\prime}, w^{\prime}\right)\right), \\
\widehat{\widehat{E}}= & \sum_{k, m=0}^{\infty} \int \ldots \int d x_{1} d y_{1} \ldots d x_{k+2 m} d y_{k+2 m} \hat{b}^{+}\left(x_{1}, y_{1}\right) \ldots \hat{b}^{+}\left(x_{k}, y_{k}\right) \times \\
& \times \widehat{B}_{1}^{+}\left(x_{k+1}, x_{k+2}\right) \widehat{B}_{2}^{+}\left(y_{k+1}, y_{k+2}\right) \ldots \widehat{B}_{1}^{+}\left(x_{k+2 m-1}, x_{k+2 m}\right) \times \\
& \times \widehat{B}_{2}^{+}\left(y_{k+2 m-1}, y_{k+2 m}\right) \times
\end{aligned}
$$




$$
\begin{aligned}
& \times \underset{x_{1}, \ldots, x_{k+2 m}}{\operatorname{Asymm}} \underset{y_{1}, \ldots, y_{k+2 m}}{\operatorname{Asymm}}\left(\hat{b}^{-}\left(x_{1}, y_{1}\right) \ldots \hat{b}^{-}\left(x_{k}, y_{k}\right) \widehat{B}_{1}^{-}\left(x_{k+1}, x_{k+2}\right) \times\right. \\
& \left.\times \widehat{B}_{2}^{-}\left(y_{k+1}, y_{k+2}\right) \ldots \widehat{B}_{1}^{-}\left(x_{k+2 m-1}, x_{k+2 m}\right) \widehat{B}_{2}^{-}\left(y_{k+2 m-1}, y_{k+2 m}\right)\right) \times \\
& \times \exp \left(-\iint d x d y \hat{b}^{+}(x, y) \hat{b}^{-}(x, y)-\iint d z d w \widehat{B}_{1}^{+}(z, w) \widehat{B}_{1}^{-}(z, w)-\right. \\
& \left.-\iint d z^{\prime} d w^{\prime} \widehat{B}_{2}^{+}\left(z^{\prime}, w^{\prime}\right) \widehat{B}_{2}^{-}\left(z^{\prime}, w^{\prime}\right)\right) .
\end{aligned}
$$

Символы операторов (13) и (16) определяют следующую систему уравнений:

$$
\begin{aligned}
i \frac{\partial}{\partial t} G\left(x_{1}, x_{2}\right)= & \int d z\left(T\left(x_{2}, z\right) G\left(x_{1}, z\right)-T\left(z, x_{1}\right) G\left(z, x_{2}\right)\right)+ \\
& +\iiint d y d z d w V\left(x_{2}, y ; z, w\right)\left(G\left(x_{1}, z\right) G(y, w)-\right. \\
& \left.-G\left(x_{1}, w\right) G(y, z)-R^{*}\left(x_{1}, y\right) R(w, z)\right)- \\
& -\iiint d y d z d w V\left(w, z ; x_{1}, y\right)\left(G\left(z, x_{2}\right) G(w, y)-\right. \\
& \left.-G\left(w, x_{2}\right) G(z, y)-R\left(y, x_{2}\right) R^{*}(z, w)\right), \\
i \frac{\partial}{\partial t} R\left(x_{1}, x_{2}\right)= & \int d z\left(T\left(x_{2}, z\right) R\left(x_{1}, z\right)+\right. \\
& \left.+T\left(x_{1}, z\right) R\left(z, x_{2}\right)\right)+\iint d z d w V\left(x_{1}, x_{2} ; z, w\right) R(z, w)+ \\
& +\iiint d y d z d w V\left(x_{2}, y ; z, w\right)\left(G(y, w) R\left(x_{1}, z\right)-\right. \\
& \left.-G(y, z) R\left(x_{1}, w\right)-G\left(y, x_{1}\right) R(w, z)\right)+ \\
& +\iiint d y d z d w V\left(x_{1}, y ; w, z\right)\left(G(y, w) R\left(z, x_{2}\right)-\right. \\
& \left.-G(y, z) R\left(w, x_{2}\right)-G\left(y, x_{2}\right) R(z, w)\right) \\
G(x, y)= & G^{*}(y, x), \quad R(x, y)=-R(y, x) .
\end{aligned}
$$

При $R=0$ система уравнений (32), (33) сводится к уравнению (29). Уравнения (32), (33) хорошо известны в теории сверхпроводимости БКШ. Рассмотрим в пространстве $L_{2}(\mathbf{T})$ операторы $\widehat{H}, \widehat{B}, \widehat{G}$ и $\widehat{R}$, ядра которых соответственно суть

$$
\begin{aligned}
& H(x, y)=T(x, y)+\iint d z d w(V(x, z ; y, w)-V(x, z ; w, y)) G(z, w), \\
& B(x, y)=\iint d z d w V(x, y ; z, w) R(z, w)
\end{aligned}
$$

$G(x, y)$ и $R(x, y)$. Система уравнений $(32),(33)$ может быть записана в виде

$$
i \frac{\partial}{\partial t} \hat{A}=[\hat{A}, \hat{L}]
$$


где $\hat{A}$ и $\hat{L}$ - следуюшие операторы в $L_{2}(\mathbf{T}) \oplus L_{2}(\mathbf{T})$ :

$$
\hat{A}=\left(\begin{array}{cc}
\widehat{G}-\frac{1}{2} & -\widehat{R}^{*} \\
\widehat{R}^{2} & -\widehat{G}^{*}+\frac{1}{2}
\end{array}\right), \quad \hat{L}=\left(\begin{array}{cc}
\widehat{H}^{*} & -\widehat{B}^{*} \\
\widehat{B} & -\widehat{H}
\end{array}\right) .
$$

Решения уравнения (34), не зависяшие от $t$, могут быть получены из следуюшего самосогласованного ( $\widehat{H}$ и $\widehat{B}$ зависят от $\widehat{G}$ и $\widehat{R})$ уравнения:

$$
\hat{A}=f(\hat{L}),
$$

где $f$-произвольная функция. Для определения свободной энергии системы фермионов можно применить "пуантилистическую" картину, из которой следует, что $f(\xi)$ в $(36)$ имеет вид $f(\xi)=\left(e^{\xi / \theta}+1\right)^{-1}$. Теперь интегральное уравнение совпадает с температурными уравнениями БКШ-Боголюбова. Из них определяются температура фазового перехода из сверхпроводящего состояния в нормальное и скачок теплоемкости при этом переходе. Таким образом, ультравторичное квантование и отвечающий ему символ (полагаем операторы $c$-числами) позволяют получить известные уравнения непосредственно. Учет квантованной энтропии позволяет получить нечто большее - зависимость температуры фазового перехода от тока.

\section{4. “ДУ́ХИ"}

4.1. Вторично-квантованная энтропия. В качестве примера рассмотрим систему из $N$ тождественных бозонов. Поместим ее на трехмерный тор $\mathbf{T}_{1}$, длина сторон которого равна $L$. Оператор Гамильтона этой системы действует в пространстве симметричных функций из $L_{2}\left(\mathbf{T}_{1}^{N}\right)$; предположим, что он имеет вид

$$
H_{1 N}=\sum_{j=1}^{N} \mathcal{E}\left(-i \hbar \frac{\partial}{\partial x_{j}}\right),
$$

где $x_{j}=\left(x_{j}^{1}, x_{j}^{2}, x_{j}^{3}\right) \in \mathbf{T}_{1}, \hbar>0$ - постоянная Планка, $\mathcal{E}(p) \in C^{\infty}\left(\mathbb{R}^{3}\right)$ - функция, для которой справедливо равенство

$$
\mathcal{E}\left(\frac{2 \pi \hbar}{L} G l+\frac{2 \pi \hbar}{L} n\right)=\mathcal{E}\left(\frac{2 \pi \hbar}{L} G l\right),
$$

где $l=\left(l_{1}, l_{2}, l_{3}\right), l_{1}, l_{2}, l_{3}$ - целые числа, $G>1$ - целое число, $n=\left(n_{1}, n_{2}, n_{3}\right), 0 \leqslant$ $n_{1}, n_{2}, n_{3}<G$ - целые числа.

Введем тор $\mathbf{T}_{2}$, длина сторон которого равна $L / G$. Рассмотрим пространство симметричных функций из $L_{2}\left(\mathbf{T}_{2}^{N}\right)$. Введем в этом пространстве оператор $H_{2 N}(\theta)$, зависящий от параметра $\theta>0$. Рассмотрим полную систему функций вида

$$
\Psi_{\{q\}}\left(y_{1}, \ldots, y_{N}\right)=\underset{y_{1}, \ldots, y_{N}}{\operatorname{Symm}} e^{\frac{i}{\hbar}\left(q_{1} y_{1}+\cdots+q_{N} y_{N}\right)},
$$


где $\{q\}$ - набор векторов $q_{1}, q_{2}, \ldots, q_{N}$, каждый из которых имеет вид $q=(2 \pi \hbar / L) G$. $\left(n_{1}, n_{2}, n_{3}\right), n_{1}, n_{2}, n_{3}$ - целые числа, $y_{j} \in \mathbf{T}_{2}, \operatorname{Symm}_{y_{1}, \ldots, y_{N}}$ - оператор симметризации относительно переменных $y_{1}, \ldots, y_{N}$. На функции (39) оператор $H_{2 N}$ действует следуюшим образом:

$$
\left(H_{2 N} \Psi_{\{q\}}\right)\left(y_{1}, \ldots, y_{N}\right)=\left(\sum_{q} \mathcal{E}(q) N_{q}-\theta \sum_{q} \ln \frac{\left(N_{q}+G^{3}-1\right) !}{N_{q} !\left(G^{3}-1\right) !}\right) \Psi_{\{q\}}\left(y_{1}, \ldots, y_{N}\right),
$$

где $q=(2 \pi \hbar / L) G \cdot\left(n_{1}, n_{2}, n_{3}\right), \sum_{q}$ - обозначение для суммы по множеству всех таких векторов $q, N_{q}$ - целые числа, зависяшие от $q$ и от набора $\{q\}$ :

$$
N_{q}=\sum_{s=1}^{N} \delta_{q q_{s}}
$$

где $\delta_{q q^{\prime}}=1$ при $q=q^{\prime}$ и $\delta_{q q^{\prime}}=0$ при $q \neq q^{\prime}$.

Имеет место следуюшая

Лемма 1. Справедливо равенство

$$
\operatorname{Sp} e^{-\frac{H_{1 N}}{\theta}}=\operatorname{Sp} e^{-\frac{H_{2 N}(\theta)}{\theta}} .
$$

ДоКАЗАТЕЛЬСТво. Полная система собственных функций оператора $H$ имеет вид

$$
\Psi\left(x_{1}, \ldots, x_{N}\right)=\underset{x_{1}, \ldots, x_{N}}{\operatorname{Symm}} e^{\frac{i}{\hbar}\left(p_{1} x_{1}+\cdots+p_{N} x_{N}\right)},
$$

где $\{p\}$ - набор векторов $p_{1}, \ldots, p_{N}$, каждый из которых имеет вид $p_{j}=(2 \pi \hbar / L) G$. $\left(n_{1}, n_{2}, n_{3}\right), x_{j} \in \mathbf{T}_{1}$. Собственные значения оператора $H_{1 N}$, отвечаюшие (41), суть

$$
\lambda_{\{p\}}=\sum_{j=1}^{N} \mathcal{E}\left(p_{j}\right) .
$$

В силу свойства (38) спектр (42) является вырожденным. Все собственные значения (42) могут быть представлены в виде

$$
E_{\{N\}}=\sum_{q} \mathcal{E}(q) N_{q}
$$

где $\{N\}$ - набор целых чисел $N_{q} \geqslant 0$ таких, что $\sum_{q} N_{q}=N$, причем кратность собственного значения (43) равна

$$
\Gamma_{\{N\}}=\prod_{q} \frac{\left(N_{q}+G^{3}-1\right) !}{N_{q} !\left(G^{3}-1\right) !} .
$$


По определению

$$
\operatorname{Sp} e^{-\frac{H_{1 N}}{\theta}}=\sum_{\{N\}} e^{-\frac{E_{\{N\}}}{\theta}} \Gamma_{\{N\}}
$$

но учитывая определение оператора $H_{2 N}(\theta)$ и формулы $(43),(44)$, получим, что правая часть равенства (45) есть $\operatorname{Sp} e^{-\frac{H_{2 N}(\theta)}{\theta}}$.

Рассмотрим бозонное фоковское пространство $\mathcal{H}_{1}[25]$, элементами которого являются последовательности функций $\left\{\Psi_{N}\right\}, N=0,1,2, \ldots$, где $\Psi_{N}\left(x_{1}, \ldots, x_{N}\right) \in L_{2}\left(\mathbf{T}_{1}^{N}\right)$ - функция, симметричная относительно перестановок любых пар переменных $x_{j}$ и $x_{j^{\prime}}$, $j, j^{\prime}=1,2, \ldots, N$. Введем в пространстве $\mathcal{H}_{1}$ следуюший оператор:

$$
\widehat{H}_{1}=\int d x \widehat{\psi}_{1}^{+}(x) \mathcal{E}\left(-i \hbar \frac{\partial}{\partial x}\right) \widehat{\psi}_{1}^{-}(x),
$$

где $\widehat{\psi}_{1}^{+}(x), \widehat{\psi}_{1}^{-}(x)$ - соответственно операторы рождения и уничтожения [25] в пространстве $\mathcal{H}_{1}$.

Аналогично рассмотрим бозонное фоковское пространство $\mathcal{H}_{2}$, состоящее из последовательностей функций $\left\{\Phi_{N}\right\}, N=0,1,2, \ldots$, где функция $\Phi_{N}\left(y_{1}, \ldots, y_{N}\right)$ симметрична относительно перестановок пар переменных $y_{j}$ и $y_{j^{\prime}}, j, j^{\prime}=1,2, \ldots, N$. Операторы рождения и уничтожения в $\mathcal{H}_{2}$ обозначим соответственно $\widehat{\psi}_{2}^{+}(y)$ и $\widehat{\psi}_{2}^{-}(y)$. Введем в $\mathcal{H}_{2}$ следуюшие операторы:

$$
\widehat{\psi}_{q}^{+}=\int d y\left(\frac{G}{L}\right)^{3} e^{-\frac{i}{\hbar} q y} \widehat{\psi}_{2}^{+}(y), \quad \widehat{\psi}_{q}^{-}=\int d y\left(\frac{G}{L}\right)^{3} e^{-\frac{i}{\hbar} q y} \widehat{\psi}_{2}^{-}(y),
$$

где $q=(2 \pi \hbar / L) G \cdot\left(n_{1}, n_{2}, n_{3}\right), n_{1}, n_{2}, n_{3}$ - целые числа.

Эти операторы удовлетворяют коммутационным соотношениям

$$
\left[\widehat{\psi}_{q}^{-}, \widehat{\psi}_{q^{\prime}}^{+}\right]=\delta_{q q^{\prime}}, \quad\left[\widehat{\psi}_{q}^{-}, \widehat{\psi}_{q^{\prime}}^{-}\right]=\left[\widehat{\psi}_{q}^{+}, \widehat{\psi}_{q^{\prime}}^{+}\right]=0
$$

а кроме того, на вакуумный вектор $\Phi_{0}[25]$ пространства $\mathcal{H}_{2}$ оператор действует следующим образом: $\widehat{\psi}_{q}^{-} \Phi_{0}=0$. Введем в пространстве $\mathcal{H}_{2}$ оператор

$$
\widehat{H}_{2}(\theta)=\sum_{q} \mathcal{E}(q) \widehat{\psi}_{q}^{+} \widehat{\psi}_{q}^{-}-\theta \sum_{q} \ln \left[\frac{\left.\Gamma\left(\llbracket \widehat{\psi}_{q}^{+} \widehat{\psi}_{q}^{-}\right]+G^{3}\right)}{\left.\Gamma\left(\llbracket \widehat{\psi}_{q}^{+} \widehat{\psi}_{q}^{-}\right]+1\right) \Gamma\left(G^{3}\right)}\right],
$$

где $\Gamma(t), t \in \mathbb{R},-$ гамма-функция Эйлера, $\llbracket \cdot \rrbracket$ - автономные скобки [15].

$N$-частичным подпространством пространства $\mathcal{H}_{1}$ или $\mathcal{H}_{2}$ называется [25] подпространство, элементы которого являются собственными функциями соответствующего оператора числа частищ

$$
\widehat{N}_{1}=\int d x \widehat{\psi}_{1}^{+}(x) \widehat{\psi}_{1}^{-}(x) \text { или } \widehat{N}_{2}=\int d y \widehat{\psi}_{1}^{+}(y) \widehat{\psi}_{1}^{-}(y)
$$

с собственным значением $N$.

Из леммы 1 следует

5 Теоретическая и математическая физика, т. 129, № 3, 2001 г. 
УТВЕРЖДЕНИЕ 1. Справедливо равенство

$$
\operatorname{Sp}_{N} e^{-\frac{\widehat{H}_{1}}{\theta}}=\operatorname{Sp}_{N} e^{-\frac{\widehat{H}_{2}(\theta)}{\theta}}
$$

где $\mathrm{Sp}_{N}$ обозначает след ограничения оператора на $N$-частичное подпространство.

Мы выбрали только один "дух" $G$ по размеру тора, в то время как стремится к бесконечности не только объем (тор), но и число частиц. Если делать аналогичную процедуру по числу частищ, появится другой “дух". Если же число частиц стремится к бесконечности много медленнее, чем объем, то можно ограничиться одним “духом”. Следовательно, в нашем случае $L^{3} \gg N \gg 1$, а значит и $G \gg N$. Тогда согласно формуле (48) мы получаем

$$
\widehat{F}(\theta) \stackrel{\text { def }}{=} \widehat{H}_{2}(\theta)=\sum_{q} \mathcal{E}(q) \widehat{\psi}_{q}^{+} \widehat{\psi}_{q}^{-}-\theta \sum_{q} \ln \llbracket \Gamma\left(\widehat{\psi}_{q}^{+} \widehat{\psi}_{q}^{-}\right) \rrbracket+\theta\left(\sum_{q} \widehat{\psi}_{q}^{+} \widehat{\psi}_{q}^{-}\right)(\ln G+1),
$$

и поскольку $\sum_{q} \widehat{\psi}_{q}^{+} \widehat{\psi}_{q}^{-}$есть полное число частиц, то последний член в свободной энергии является константой и “дух" $G$ не влияет на термодинамические характеристики рассматриваемой системы.

4.2. Ультравторично-квантованная энтропия для бозонов со статспином. Перейдем к рассмотрению ультравторично-квантованных задач. Рассмотрим пространство $\mathcal{F}_{1 N}$, элементами которого являются функции

$$
\Psi\left(x_{1}, s_{1} ; x_{2}, s_{2} ; \ldots ; x_{N}, s_{N}\right)
$$

где $x_{j} \in \mathbf{T}_{1}$, а $s_{j}, j=1,2, \ldots, N$, - дискретная переменная, принимающая значения $1,2, \ldots, J_{1}$. Будем называть эту переменную статистическим спином (статспином) или номером. Функции (50) симметричны относительно любых перестановок пар переменных $\left(x_{j}, s_{j}\right)$ и $\left(x_{j^{\prime}}, s_{j^{\prime}}\right)$, а также принадлежат пространству $L_{2}\left(\left(\mathbf{T}_{1} \times\left\{1,2, \ldots, J_{1}\right\}\right)^{N}\right)$, т.е. справедливо неравенство

$$
\sum_{s_{1}=1}^{J_{1}} \ldots \sum_{s_{N}=1}^{J_{1}} \int \ldots \int d x_{1} \ldots d x_{N}\left|\Psi\left(x_{1}, s_{1} ; \ldots ; x_{N}, s_{N}\right)\right|^{2}<\infty
$$

Левая часть этого неравенства равна квадрату нормы элемента $(50)$ пространства $\mathcal{F}_{1 N}$.

Введем пространство $\mathcal{F}_{1}=\bigoplus_{N=0}^{\infty} \mathcal{F}_{1 N}$, состояшее из последовательностей функций $\left\{\Psi_{N}\right\}, N=0,1,2, \ldots$, где $\Psi_{N} \in \mathcal{F}_{1 N}$ - функция вида (50). Пространство $\mathcal{F}_{1}$ является бозонным фоковским пространством, и в нем стандартным образом вводятся операторы рождения $\hat{b}_{1}^{+}(x, s)$ и уничтожения $\hat{b}_{1}^{-}(x, s)$ частиц со статспином. 
Симметричным подпространством $\mathcal{F}_{1}^{\text {Symm }}$ пространства $\mathcal{F}_{1}$ назовем множество последовательностей $\left\{\Psi_{N}\right\}, \quad N=0,1,2, \ldots$, которые состоят из функций (50), симметричных относительно любых перестановок переменных $x_{j}$ и $x_{j^{\prime}}$ и симметричных относительно любых перестановок переменных $s_{j}$ и $s_{j^{\prime}} . N$-частичное подпространство пространства $\mathcal{F}_{1}$ изометрически изоморфно пространству $\mathcal{F}_{1 N}$. Симметричным $N$-частичным подпространством $\mathcal{F}_{1 N}^{\text {Symm }}$ пространства $\mathcal{F}_{1}$ будем называть пересечение подпространств $\mathcal{F}_{1 N}$ и $\mathcal{F}_{1}^{\text {Symm }}$.

Теперь введем пространства $\mathcal{F}_{2 N}$ и $\mathcal{F}_{2}$ полностью аналогично определению пространств $\mathcal{F}_{1 N}$ и $\mathcal{F}_{1}$, заменив в этом определении переменные $x_{j} \in \mathbf{T}_{1}$ на $y_{j} \in \mathbf{T}_{2}$, а переменные $s_{j}=1, \ldots, J_{1}$ на $t_{j}=1, \ldots, J_{2}$, где $J_{2}=J_{1} / Q$ и $Q>1$ - целые числа. Кроме того, аналогично введем симметричное $\mathcal{F}_{2}^{\mathrm{Symm}}, N$-частичное $\mathcal{F}_{2 N}$ и $N$-частичное симметричное $\mathcal{F}_{2 N}^{\text {Symm }}$ подпространства пространства $\mathcal{F}_{2}$. Пространство $\mathcal{F}_{2}$, так же как и $\mathcal{F}_{1}$, является бозонным фоковским пространством; операторы рождения и уничтожения в $\mathcal{F}_{2}$ будем обозначать соответственно $\hat{b}_{2}^{+}(y, t)$ и $\hat{b}_{2}^{-}(y, t)$.

Пусть задана последовательность операторов $\left\{A_{N}\right\}, N=0,1, \ldots$, где $A_{N}$ - оператор в пространстве симметричных функций $\Psi\left(x_{1}, \ldots, x_{N}\right) \in L_{2}\left(\mathbf{T}_{1}^{N}\right)$ такой, что

$$
\left(A_{N} \Psi\right)\left(x_{1}, \ldots, x_{N}\right)=A_{N}\left(\stackrel{2}{x_{1}}, \ldots, \stackrel{2}{x}{ }_{N} ;-i \hbar \frac{\stackrel{1}{\partial}}{\partial x_{1}}, \ldots,-i \hbar \frac{\stackrel{1}{\partial}}{\partial x_{N}}\right) \Psi\left(x_{1}, \ldots, x_{N}\right) .
$$

В случае, когда коэффициенты суммирования в формулах $(3),(16)$ равны $1 / N !$, следуюший оператор в пространстве $\mathcal{F}_{1}$ называется ультравторично-квантованным:

$$
\begin{aligned}
\overline{\hat{A}}= & \sum_{N=0}^{\infty} \frac{1}{N !} \sum_{s_{1}=1}^{J_{1}} \ldots \sum_{s_{N}=1}^{J_{N}} \int \ldots \int d x_{1} \ldots d x_{N} \hat{b}_{1}^{+}\left(x_{1}, s_{1}\right) \ldots \hat{b}_{1}^{+}\left(x_{N}, s_{N}\right) \times \\
& \times A_{N} \underset{x_{1}, \ldots, x_{N}}{\operatorname{Symm}}\left(\hat{b}_{1}^{-}\left(x_{1}, s_{1}\right) \ldots \hat{b}_{1}^{-}\left(x_{N}, s_{N}\right)\right) \times \\
& \times \exp \left(-\sum_{s=1}^{J_{1}} \int d x \hat{b}_{1}^{+}(x, s) \hat{b}_{1}^{-}(x, s)\right) .
\end{aligned}
$$

С помошью непосредственного вычисления легко убедиться, что ультравторичноквантованные операторы (51) обладают следуюшими свойствами.

УТВеРЖДЕнИЕ 2. Справедливо равенство

$$
\operatorname{Sp}_{N, \operatorname{Symm}} \overline{\hat{A}}=\left(\operatorname{Sp} A_{N}\right) \frac{\left(N+J_{1}-1\right) !}{N !\left(J_{1}-1\right) !},
$$

где $\mathrm{Sp}_{N, \text { Sуmm }} \overline{\hat{A}}$ обозначает след ограничения оператора $\overline{\hat{A}}$ на $N$-частичное симметричное подпространство $\mathcal{F}_{1 N}^{\text {Symm }}$. 
УТВЕРЖДЕНИЕ 3. Пусть $\widehat{\widehat{B}}$ - ультравторично-квантованный оператор вида (51), отвечающий последовательности операторов $\left\{B_{N}\right\}, \quad N=0,1,2, \ldots$, где $B_{N}=A_{N}^{n}$, а $n>1$ - челое число. Тогда имеет место равенство

$$
\overline{\widehat{B}}=(\overline{\hat{A}})^{n} .
$$

Будем далее обозначать через $\overline{\widehat{H}}_{1}$ оператор в пространстве $\mathcal{F}_{1}$ вида $(51)$, отвечающий последовательности операторов $\left\{H_{1 N}\right\}$, где $H_{1 N}$ - операторы (37). С помошью утверждений 2 и 3 можно доказать, что имеет место

Лемма 2. Справедливо равенство

$$
\operatorname{Sp}_{N, \text { Symm }} e^{-\frac{\widehat{H}_{1}}{\theta}}=\operatorname{Sp} e^{-\frac{\widehat{H}_{1 N}}{\theta}} \frac{\left(N+J_{1}-1\right) !}{N !\left(J_{1}-1\right) !} .
$$

Введем в пространстве $\mathcal{F}_{2}$ следуюшие операторы:

$$
\hat{b}_{q t}^{-}=\frac{1}{L^{\frac{3}{2}}} \int d y \hat{b}_{2}^{-}(y, t) e^{-\frac{i q}{\hbar} y}, \quad \hat{b}_{q t}^{+}=\frac{1}{L^{\frac{3}{2}}} \int d y \hat{b}_{2}^{+}(y, t) e^{\frac{i q}{\hbar} y} .
$$

Эти операторы удовлетворяют коммутационным соотношениям

$$
\left[b_{q t}^{-}, b_{q^{\prime} t^{\prime}}^{+}\right]=\delta_{q q^{\prime}} \delta_{t t^{\prime}}, \quad\left[b_{q t}^{ \pm}, b_{q^{\prime} t^{\prime}}^{ \pm}\right]=0,
$$

и вакуумный вектор операторов (54) совпадает с вакуумным вектором операторов $\hat{b}_{2}^{ \pm}(y, t)$.

Рассмотрим в пространстве $\mathcal{F}_{2}$ операторы $\overline{\widehat{H}}_{2}(\theta)$ и $\overline{\widehat{F}}(\theta)$ вида

$$
\begin{aligned}
\overline{\mathrm{H}}_{2}(\theta)= & \sum_{N=0}^{\infty} \frac{1}{N !} \sum_{q_{1}} \cdots \sum_{q_{N}} \sum_{t_{1}=1}^{J_{2}} \cdots \sum_{t_{N}=1}^{J_{2}} \hat{b}_{q_{1} t_{1}}^{+} \ldots \hat{b}_{q_{N} t_{N}}^{+} \underset{q_{1}, \ldots, q_{N}}{\operatorname{Symm}}\left(\hat{b}_{q_{1} t_{1}}^{-} \ldots \hat{b}_{q_{N}}^{-} t_{N}\right) \times \\
& \times\left(\sum_{q} \mathcal{E}(q) M_{q}-\theta \sum_{q} \ln \left[\frac{\left(M_{q}+G^{3}-1\right) !}{M_{q} ! G^{3} !}\right]-\theta \sum_{t=1}^{J_{2}} \ln \left[\frac{\left(K_{t}+Q-1\right) !}{K_{t} !(Q-1) !}\right]\right) \times \\
& \times \exp \left(-\sum_{q} \sum_{t=1}^{J_{2}} \hat{b}_{q t}^{+} \hat{b}_{q t}^{-}\right), \\
\overline{\widehat{F}}(\theta)= & \left.\sum_{q} \sum_{t=1}^{J_{2}} \mathcal{E}(q) \hat{b}_{q t}^{+} \hat{b}_{q t}^{-}+\theta \sum_{q} \sum_{t=1}^{J_{2}} \ln \left[\Gamma\left(\llbracket \llbracket \hat{b}_{q t}^{+} \hat{b}_{q t}^{-}\right]+1\right)\right]-\theta \sum_{q} \ln \left[\frac{\Gamma\left(\widehat{M}_{q}+G^{3}\right)}{\Gamma\left(G^{3}\right)}\right]- \\
& -\theta \sum_{t=1}^{J_{2}} \ln \left[\frac{\Gamma\left(\widehat{K}_{t}+Q\right)}{\Gamma(Q)}\right]-\theta J_{1} \ln J_{1}+\theta\left(N+J_{1}\right) \ln \left(N+J_{1}\right)-\theta N,
\end{aligned}
$$

где в формуле (55) величины $M_{q}$ и $K_{t}$ являются функциями от $q_{1}, \ldots, q_{N}$ и $t_{1}, \ldots, t_{N}$ :

$$
M_{q}=\sum_{\alpha=1}^{N} \delta_{q q_{\alpha}}, \quad K_{t}=\sum_{\alpha=1}^{N} \delta_{t t_{\alpha}},
$$

а в формуле (56) операторы $\widehat{M}_{q}$ и $\widehat{K}_{t}$ имеют вид

$$
\widehat{M}_{q}=\sum_{t=1}^{J_{2}} \hat{b}_{q t}^{+} \hat{b}_{q t}^{-}, \quad \widehat{K}_{t}=\sum_{q} \hat{b}_{q t}^{+} \hat{b}_{q t}^{-} .
$$

Справедлива следуюшая 
УЛЬТРАВТОРИЧНОЕ КВАНТОВАНИЕ И “ДУ́ХИ” В КВАНТОВАННОЙ ЭНТРОПИИ 485

Лемма 3. Справедливо равенство

$$
\operatorname{Sp}_{N, \text { Symm }} e^{-\frac{\overline{\widehat{H}}_{1}}{\theta}}=\operatorname{Sp}_{N, \text { Symm }} e^{-\frac{\overline{\widehat{H}}_{2}(\theta)}{\theta}}=\operatorname{Sp}_{N} e^{-\frac{\overline{\widehat{F}}(\theta)}{\theta}} .
$$

Доказательство этой леммы аналогично доказательству леммы 1.

Рассмотрим теперь случай, когда $L \rightarrow \infty$ и $N \rightarrow \infty$ так, что $N / L^{3}=n=$ const (термодинамический предел).

Свободной энергией при температуре $\theta>0$ системы $N$ бозонов с гамильтонианом $H_{1 N}(37)$ называют величину

$$
F(\theta)=-\theta \ln \left(\operatorname{Sp} e^{-\frac{H_{1 N}}{\theta}}\right) .
$$

Пусть $G \sim L^{\varepsilon}, 0<\varepsilon<1$, тогда имеет место

ПреДЛОЖенИЕ 2. При $N \rightarrow \infty, \quad G \rightarrow \infty, L \rightarrow \infty$ свободная әнергия (59) асимптотически равна минимальному собственному значению оператора $\widehat{H}_{2}(\theta)$ (48) на $N$-частичном подпространстве пространства $\mathcal{H}_{2}$.

Приведем эвристическое обоснование. Из леммы 1 следует, что

$$
F(\theta)=-\theta \ln \left(\operatorname{Sp}_{N} e^{-\frac{\widehat{H}_{2}(\theta)}{\theta}}\right) .
$$

Собственные значения оператора $\widehat{H}_{2}(\theta)(48)$ на $N$-частичном подпространстве пространства $\mathcal{H}_{2}$ имеют вид

$$
E_{\{N\}}(\theta)=\sum_{q} \mathcal{E}(q) N_{q}-\theta \sum_{q} \ln \left[\frac{\left(N_{q}+G^{3}-1\right) !}{N_{q} !\left(G^{3}\right) !}\right],
$$

где $\{N\}$ - набор целых чисел $N_{q} \geqslant 0$ такой, что

$$
\sum_{q} N_{q}=N
$$

По определению следа

$$
\operatorname{Sp}_{N} e^{-\frac{\widehat{H}_{2}(\theta)}{\theta}}=\sum_{\{N\}} e^{-\frac{E_{\{N\}}(\theta)}{\theta}},
$$

где $\sum_{\{N\}}$ обозначает суммирование по всем наборам целых чисел $\{N\}$ таких, что выполняется (61).

Векторы $q$ в выражениях (60) и (61) пробегают множество значений $(2 \pi \hbar / L) G \times$ $\left(n_{1}, n_{2}, n_{3}\right)$, где $n_{1}, n_{2}, n_{3}$ - целые числа. Поэтому сделаем в выражениях (60)-(62) замену $n(q)=N_{q} / G^{3}$. Из условия (61) следует, что $n(q) \sim N / L^{3}$. При такой замене выражение (60) асимптотически равно

$$
E_{\{N\}} \approx \sum_{q} G^{3} n(q)+\theta \sum_{q} G^{3} n(q) \ln n(q)-\theta \sum_{q} G^{3}(1+n(q)) \ln (1+n(q)) .
$$


В выражении (63) при всех членах стоит большой параметр $G^{3}$, поэтому для вычисления суммы в правой части равенства (62) можно воспользоваться методом Лапласа для сумм, а этот метод приводит к асимптотическому равенству

$$
\ln \left(\operatorname{Sp}_{N} e^{-\frac{\widehat{H}_{2}(\theta)}{\theta}}\right) \sim-\frac{1}{\theta} \min _{\{N\}} E_{\{N\}}(\theta),
$$

которое и требуется доказать.

В случае ультравторичного квантования справедливо аналогичное

ПреДЛОЖенИЕ 3. При $N \rightarrow \infty, G \rightarrow \infty, L \rightarrow \infty$ классическая свободная әнергия (59) асимптотически равна минимальному собственному значению оператора квантованной свободной әнергии $\overline{\widehat{F}}(\theta)(56)$.

$\mathrm{C}$ помошью лемм 2 и 3 предложение 3 доказывается так же, как и предыдущее. Заметим теперь, что если $J_{2}=1$, то пространство $\mathcal{F}_{2}$ совпадает с пространством $\mathcal{H}_{2}$, и можно сказать, что оператор $\overline{\widehat{F}}(\theta)(56)$ в этом случае совпадает с $\widehat{H}_{2}(\theta)$ (48). Таким образом, утверж дение предложения 3 в этом случае совпадает с утверждением предложения 2 .

4.3. Учет операторов рождения пар в ультравторично-квантованной энтропии. Аналогично тому, как в предыдуших пунктах была получена свободная энергия во вторично-квантованном и ультравторично-квантованном "холостом" случаях, можно получить свободную энергию для системы, в которой есть конденсат парных частиц. В этом случае аналогично вводится пространство $\mathcal{F}$.

Ультравторично-квантованные операторы (51) с операторами рождения $\widehat{B}^{+}(x, y)$ и уничтожения $\widehat{B}^{-}(x, y)$ пар принимают вид

$$
\begin{aligned}
\overline{\hat{A}}= & \sum_{k, m=0}^{\infty} \sum_{s_{1}=1}^{J_{1}} \ldots \sum_{s_{k}=1}^{J_{k}} \int \ldots \int d x_{1} \ldots d x_{k} d y_{1} \ldots d y_{2 m} \times \\
& \times \hat{b}^{+}\left(x_{1}, s_{1}\right) \ldots \hat{b}^{+}\left(x_{k}, s_{k}\right) \widehat{B}^{+}\left(y_{1}, y_{2}\right) \ldots \widehat{B}^{+}\left(y_{2 m-1}, y_{2 m}\right) \times \\
& \times A_{k+2 m} \underset{x_{1}, \ldots, x_{k}, y_{1}, \ldots, y_{2 m}}{\operatorname{Symm}}\left(\hat{b}^{-}\left(x_{1}, s_{1}\right) \ldots \hat{b}^{-}\left(x_{k}, s_{k}\right) \times\right. \\
& \left.\times \widehat{B}^{-}\left(y_{1}, y_{2}\right) \ldots \widehat{B}^{-}\left(y_{2 m-1}, y_{2 m}\right)\right) \times \\
& \times \exp \left(-\sum_{s=1}^{J} \int d x \hat{b}^{+}(x, s) \hat{b}^{-}(x, s)-\iint d y d z \widehat{B}^{+}(y, z) \widehat{B}^{-}(y, z)\right) .
\end{aligned}
$$

Для таких операторов справедливо утверждение, аналогичное утверждению 2. А именно, справедливо равенство

$$
\operatorname{Sp}_{k, m, \text { Symm }} \overline{\hat{A}}=\left(\operatorname{Sp} A_{N}\right) \frac{\left(k+J_{1}-1\right) !}{k !\left(J_{1}-1\right) !} .
$$


Кроме того, в случае с парами также справедливо утверждение 3. Введем операторы

$$
\begin{aligned}
& \widehat{B}_{q_{1} q_{2}}^{-}=\iint d y_{1} d y_{2} \widehat{B}^{-}\left(y_{1}, y_{2}\right) e^{-i \frac{q_{1} y_{1}+q_{2} y_{2}}{\hbar}} \\
& \widehat{B}_{q_{1} q_{2}}^{+}=\iint d y_{1} d y_{2} \widehat{B}^{+}\left(y_{1}, y_{2}\right) e^{i \frac{q_{1} y_{1}+q_{2} y_{2}}{\hbar}}
\end{aligned}
$$

которые удовлетворяют коммутационным соотношениям

$$
\left[\widehat{B}_{q_{1} q_{2}}^{-}, \widehat{B}_{q_{1}^{\prime} q_{2}^{\prime}}^{+}\right]=\delta_{q_{1} q_{2}} \delta_{q_{1}^{\prime} q_{2}^{\prime}}, \quad\left[\widehat{B}_{q_{1} q_{2}}^{ \pm}, \widehat{B}_{q_{1}^{\prime} q_{2}^{\prime}}^{ \pm}\right]=0 .
$$

Рассмотрим оператор

$$
\begin{aligned}
\overline{\widehat{F}}(\theta)= & \sum_{q} \sum_{t=1}^{J_{2}} \mathcal{E}(q) \hat{b}_{q t}^{+} \hat{b}_{q t}^{-}+\sum_{q, q^{\prime}}\left(\mathcal{E}(q)+\mathcal{E}\left(q^{\prime}\right)\right) \widehat{B}_{q q^{\prime}}^{+} \widehat{B}_{q q^{\prime}}^{-}+\theta \sum_{q} \sum_{t=1}^{J_{2}} \ln \Gamma\left(\hat{b}_{q t}^{+} \hat{b}_{q t}^{-}+1\right)+ \\
& +\theta \sum_{q, q^{\prime}} \ln \Gamma\left(\widehat{B}_{q q^{\prime}}^{+} \widehat{B}_{q q^{\prime}}^{-}+1\right)-\theta \sum_{q} \ln \left[\frac{\Gamma\left(\widehat{M}_{q}+G^{3}\right)}{\Gamma\left(G^{3}\right)}\right]-\theta \sum_{t=1}^{J_{2}} \ln \left[\frac{\Gamma\left(\widehat{K}_{t}+Q\right)}{\Gamma(Q)}\right]- \\
& -\theta \ln \left[\frac{k ! m !\left(J_{1}-1\right) !}{\left(k+J_{1}-1\right) !(k+2 m) !}\right]
\end{aligned}
$$

где

$$
\widehat{M}_{q}=\sum_{t=1}^{J_{2}} \hat{b}_{q t}^{+} \hat{b}_{q t}^{-}+\sum_{q^{\prime}} \widehat{B}_{q q^{\prime}}^{+} \widehat{B}_{q q^{\prime}}^{-}+\sum_{q^{\prime}} \widehat{B}_{q^{\prime} q}^{+} \widehat{B}_{q^{\prime} q}^{-}, \quad \widehat{K}_{t}=\sum_{q} \hat{b}_{q t}^{+} \hat{b}_{q t}^{-} .
$$

В парном случае имеет место

ПРЕДЛОЖенИЕ 4. При $G \rightarrow \infty, L \rightarrow \infty, \quad N \rightarrow \infty$ классическая свободная әнергия (59) асимптотически равна минимальному собственному значению оператоpa $\widehat{\widehat{F}}(\theta)(67)$.

Обоснование предложения 4 полностью аналогично обоснованию предложения 2.

ЗАМЕчАниЕ 1. Выражение для энтропии, полученное вьше, справедливо лишш при $N \ll G^{3} \sim Q$, возможно (гипотеза!) и для конечных $N$. Это значит, что формула (67) и другие сушественно упростятся, когда мы применим формулу Стирлинга к членам, содержашим $G^{3}$ и $Q$.

В дальнейшем в определении квантованной энтропии мы будем для простоты опускать последний член в (67). В нижеследуюших формулах стирается грань между бозонами и фермионами, и они практически определяются для классических частиц. Итак,

$$
\begin{aligned}
\overline{\widehat{S}}= & \sum_{q} \sum_{t=1}^{J_{2}} \ln \left[\Gamma\left(\hat{b}_{q t}^{+} \hat{b}_{q t}^{-}+1\right)\right]+\sum_{q, q^{\prime}} \ln \left[\Gamma\left(\widehat{B}_{q q^{\prime}}^{+} \widehat{B}_{q q^{\prime}}^{-}+1\right)\right]- \\
& -\sum_{q} \ln \left[\Gamma\left(\widehat{M}_{q}\right)\right]-\sum_{t=1}^{J_{2}} \ln \left[\Gamma\left(\widehat{K}_{t}\right)\right]+\sum_{q} \widehat{M}_{q}\left(\ln G^{3}+1\right)+\sum_{t=1}^{J_{2}} \widehat{K}_{t}(\ln Q+1) .
\end{aligned}
$$

Поскольку $\sum_{q} \widehat{M}_{q}$ и $\sum_{t=1}^{J_{2}} \widehat{K}_{t}$ инварианты, то члены с “ду́хами” не играют роли. 


\section{5. ПУАНТИЛИСТИЧЕСКОЕ ОПРЕДЕЛЕНИЕ ОПЕРАТОРА ВНУТРЕННЕЙ ЭНЕРГИИ}

Внутренняя энергия термодинамической системы выражается через свободную энергию известным образом: $\mathcal{E}(\theta, V, N)=F(\theta, V, N)-\theta(\partial F(\theta, V, N) / \partial \theta)$. Поскольку введен оператор свободной энергии, то естественно ввести и оператор внутренней энергии. Сделаем это с помощью пуантилистического подхода. Пусть гамильтониан системы $N$ фермионов имеет вид (20). Рассмотрим функции $G(x, y)$ и $R(x, y)$, которые являются решением уравнения $(36)$ при $f(\xi)=\left(e^{\xi / \theta}+1\right)^{-1}-1 / 2$, где $\theta$ - температура. Введем в пространстве $\mathcal{F}$ следуюший вектор:

$$
\Psi_{\theta}=\overline{\widehat{E}} \exp \left(\sum_{s=1}^{\infty} \int d x \varphi(x, s) \hat{b}^{+}(x, s)+\frac{1}{2} \iint d y d z \Phi(y, z) \widehat{B}^{+}(y, z)\right) \Psi_{0},
$$

где функции $\varphi(x, s), \Phi(y, z)$ определяются из уравнений

$$
\begin{aligned}
\widehat{G}= & \left(1+\hat{A}^{\operatorname{tr}}\right)^{-1}\left(\hat{A}^{\operatorname{tr}}-\widehat{C}^{*}(1+\hat{A})^{-1} \widehat{C}\left(1+\hat{A}^{\mathrm{tr}}\right)^{-1} \times\right. \\
& \left.\times\left(1-\widehat{C}^{*}(1+\hat{A})^{-1} \widehat{C}\left(1+\hat{A}^{\mathrm{tr}}\right)^{-1}\right)^{-1}\right) \\
\widehat{R}= & -(1+\hat{A})^{-1} \widehat{C}\left(1+\hat{A}^{\operatorname{tr}}\right)^{-1}\left(1-\widehat{C}^{*}(1+\hat{A})^{-1} \widehat{C}\left(1+\hat{A}^{\mathrm{tr}}\right)^{-1}\right)^{-1},
\end{aligned}
$$

$\widehat{G}, \widehat{R}, \hat{A}, \widehat{C}$ - следуюшие операторы в $L_{2}\left(\mathbb{R}^{3}\right)$ :

$$
\begin{array}{ll}
(\widehat{G} f)(x)=\int d y G(x, y) f(y), & (\widehat{R} f)(x)=\int d y R(x, y) f(y), \\
(\hat{A} f)(x)=\sum_{s=1}^{\infty} \int d y \varphi(x, s) \varphi^{*}(y, s) f(y), & (\widehat{C} f)(x)=\int d y \Phi(x, y) f(y),
\end{array}
$$

а кроме того, в уравнениях (70) знаки $*$ и $\operatorname{tr}$ обозначают соответственно комплексное сопряжение и транспонирование оператора. В случае, когда система находится на трехмерном торе и гамильтониан (20) коммутирует с оператором импульса, функции $\varphi(x, s)$, $\Phi(y, z)$ представляются в виде

$$
\varphi(x, s)=\frac{\sqrt{a_{p_{s}}} e^{i p_{s} x}}{L^{\frac{3}{2}}}, \quad a_{p}=a_{-p}, \quad \Phi(x, y)=\frac{1}{L^{3}} \sum_{p} \phi_{p} e^{i p(x-y)}, \quad \phi_{p}=-\phi_{-p},
$$

где $L$ - длина сторон тора, $p$ - импульс на торе, принимающий значения на кубической решетке, $p_{s}$ - взаимно однозначное отображение множества номеров $s$ на множество значений импульса. Для вектора (69) среднее значение оператора числа пар

$$
\iint d x d y \widehat{B}^{+}(x, y) \widehat{B}^{-}(x, y)
$$


равно

$$
M=\frac{1}{2} \sum_{p} \frac{\phi_{p}^{*} \phi_{p}}{\left(1+a_{p}\right)^{2}+\phi_{p}^{*} \phi_{p}},
$$

а среднее значение оператора числа частиц с номером $s$

$$
\iint d x d y \hat{b}^{+}(x, s) \hat{b}^{-}(x, s)
$$

равно

$$
k_{s}=\frac{a_{p_{s}}\left(1+a_{p_{s}}\right)}{\left(1+a_{p_{s}}\right)^{2}+\phi_{p_{s}}^{*} \phi_{p_{s}}} .
$$

Выделим в пространстве $\mathcal{F}$ линейное подпространство $\mathcal{F}_{\theta}$ такое, что базисом в нем являются векторы, образуюшие серию собственных векторов ультравторично-квантованного гамильтониана $\widehat{\widehat{H}}$, для которых разность средних значений (73) оператора числа пар и средних значений (74) операторов числа частиц с номерами и выражений (73) и (74), соответственно, по модулю не превьшает некоторой постоянной в термодинамическом пределе величины. Понятие серии было введено и подробно разъяснено в работе [11], здесь заметим только, что для любого вектора из $\mathcal{F}_{\theta}$ и любого вектора из дополнения к $\mathcal{F}_{\theta}$ матричные элементы широкого класса операторов (этот класс содержит ультравторично-квантованные гамильтониан $\widehat{\widehat{H}}$ и единичный оператор $\overline{\widehat{E}}$ ) стремятся к нулю в термодинамическом пределе. В силу этого будем рассматривать уравнение

$$
\widehat{\widehat{P}}_{\theta} \overline{\widehat{H}}_{\widehat{P}_{\theta}} \Psi=\lambda \widehat{\widehat{P}}_{\theta} \overline{\widehat{E}}_{\widehat{P}_{\theta}} \Psi
$$

где $\widehat{\widehat{P}}_{\theta}$ - оператор ортогонального проектирования на подпространство $\mathcal{F}_{\theta}$.

ОПредЕлЕниЕ 5 . Значения $\lambda$, при которых сушествует решение уравнения (75) такое, что $\widehat{P}_{\theta} \widehat{E}_{\vec{P}} \widehat{P}_{\theta} \Psi \neq 0$, будем называть спектром проквантованной внутренней энергии при температуре $\theta$. Минимальное значение $\lambda$ будем называть квантовым значением внутренней энергии термодинамической системы при температуре $\theta$. Оператором внутренней энергии будем называть ограничение гамильтониана $\widehat{\widehat{H}}$ на подпространство $\mathcal{F}_{\theta}$ : $\widehat{P}_{\theta} \widehat{\widehat{H}}_{\widehat{P}_{\theta}}$.

Поскольку $\Psi_{\theta} \in \mathcal{F}_{\theta}$, то выражение $\left(\Psi_{\theta}, \overline{\widehat{H}} \Psi_{\theta}\right) /\left(\Psi_{\theta}, \widehat{\widehat{E}} \Psi_{\theta}\right)$ является оценкой сверху для квантового значения внутренней энергии. Это выражение для вектора (69) может быть вычислено в явном виде и совпадает с выражением для внутренней энергии в теории БКШ

В заключение заметим, что определение оператора внутренней энергии для бозонов полностью аналогично, за исключением того, что в формуле (69) функции $\varphi(x, s)$, $\Phi(y, z)$ определяются не из уравнений $(70)$, а из уравнений

$$
\begin{aligned}
\widehat{G}= & \left(1-\hat{A}^{\mathrm{tr}}\right)^{-1}\left(\hat{A}^{\mathrm{tr}}-\widehat{C}^{*}(1-\hat{A})^{-1} \widehat{C}\left(1-\hat{A}^{\mathrm{tr}}\right)^{-1} \times\right. \\
& \left.\times\left(1-\widehat{C}^{*}(1-\hat{A})^{-1} \widehat{C}\left(1-\hat{A}^{\mathrm{tr}}\right)^{-1}\right)^{-1}\right), \\
\widehat{R}= & -(1-\hat{A})^{-1} \widehat{C}\left(1-\hat{A}^{\mathrm{tr}}\right)^{-1}\left(1-\widehat{C}^{*}(1-\hat{A})^{-1} \widehat{C}\left(1-\hat{A}^{\mathrm{tr}}\right)^{-1}\right)^{-1},
\end{aligned}
$$


где $\widehat{G}, \widehat{R}, \hat{A}, \widehat{C}$ выражаются формулами (71), а функции $G(x, y), R(x, y)$ являются решениями бозонного аналога уравнения (36). Кроме того, в бозонном случае имеет место представление, аналогичное (72), в котором условие $\phi_{p}=-\phi_{-p}$ заменяется на условие $\phi_{p}=\phi_{-p}$. А также формулы для среднего числа пар (73) и среднего числа частиц с номерами (74) должны быть заменены соответственно на формулы

$$
M=\frac{1}{2} \sum_{p} \frac{\phi_{p}^{*} \phi_{p}}{\left(1-a_{p}\right)^{2}-\phi_{p}^{*} \phi_{p}}, \quad k_{s}=\frac{a_{p_{s}}\left(1-a_{p_{s}}\right)}{\left(1-a_{p_{s}}\right)^{2}-\phi_{p_{s}}^{*} \phi_{p_{s}}} .
$$

Благодарности. В заключение автор выражает глубокую благодарность Г. В. Ковалю и Е. Д. Ким за существенную помошь.

\section{Список литературы}

[1] В. П. Маслов. ТМФ. 1994. Т. 101. № 3. С. 433.

[2] В. П. Маслов. Функц. анализ и его прилож. 1994. Т. 28. № 4. С. 28.

[3] В. П. Маслов. Матем. заметки. 1994. Т. 56. № 3. С. 115.

[4] В. П. Маслов. Докл. РАН. 1995. Т. 340. № 2. С. 175.

[5] В. П. Маслов. Квантование термодинамики и ультравторичное квантование. Ижевск: НИЦ "Регулярная и хаотическая динамика", УРСС, 2000.

[6] В. П. Маслов. Матем. заметки. 1997. Т. 61. № 4. С. 627.

[7] В. П. Маслов. Матем. заметки. 1997. Т. 62. № 4. С. 633.

[8] В. П. Маслов. Матем. заметки. 1998. Т. 63. № 4. С. 635.

[9] В. П. Маслов. Матем. заметки. 1998. Т. 63. № 5. С. 792.

[10] В. П. Маслов. Матем. заметки. 1998. Т. 64. № 3. С. 470.

[11] V. P. Maslov. Russ. J. Math. Phys. 1997. V. 5. № 4. P. 473.

[12] В. П. Маслов. Докл. РАН. 1999. Т. 369. № 3. С. 320.

[13] В. П. Маслов. Докл. РАН. 1999. Т. 369. № 5. С. 607.

[14] В. П. Маслов. Докл. РАН. 1999. Т. 369. № 4. С. 466.

[15] В. П. Маслов. Матем. заметки. 1999. Т. 66. № 4. С. 630.

[16] В. П. Маслов. Матем. заметки. 1999. Т. 66. № 5. С. 706.

[17] В. П. Маслов. Матем. заметки. 1999. Т. 66. № 6. С. 701.

[18] В. П. Маслов. ТМФ. 1999. Т. 121. № 3. С. 492.

[19] В. П. Маслов. Функц. анализ и его прилож. 1999. Т. 33. № 4. С. 50.

[20] В. П. Маслов. Функц. анализ и его прилож. 2000. Т. 34. № 4. С. 35.

[21] В. П. Маслов. Вестн. МГУ. Физика. Астрономия. 2000. № 6. С. 3.

[22] В. П. Маслов. ТМФ. 2000. Т. 125. № 2. С. 297.

[23] В. П. Маслов, А. М. Чеботарев. О втором члене логарифмической асимптотики функциональных интегралов. В сб.: Итоги науки и техники. Т. 19. Теория вероятностей. Математическая статистика. Теоретическая кибернетика. Ред. Р. В. Гамкрелидзе. М.: ВИНИТИ, 1982. C. 127.

[24] Л. Д. Ландау, Е. М. Лифииц. Статистическая физика. М.: Наука, 1995.

[25] Ф. А. Березин. Метод вторичного квантования. М.: Наука, 1965.

[26] В. П. Маслов. Операторные методы. М.: Наука, 1973. 